\title{
Anticoagulation Favors Thrombus Recanalization and Survival in Patients With Liver Cirrhosis and Portal Vein Thrombosis: Results of a Meta- Analysis
}

Le Wang $\cdot$ Xiaozhong Guo $\cdot$ Xiangbo Xu $\cdot$ Valerio De Stefano

Aurelie Plessier $\cdot$ Carlos Noronha Ferreira $\cdot$ Xingshun Qi (C)

Received: September 23, 2020 / Accepted: October 23, 2020 / Published online: November 5, 2020

(c) The Author(s) 2020

\section{ABSTRACT}

Introduction: Benefit and risk of anticoagulation in cirrhotic patients with portal vein thrombosis (PVT) remain controversial, especially in those with asymptomatic PVT and in non-liver transplant candidates. Furthermore, the predictors of portal vein recanalization and bleeding events after anticoagulation are critical for making clinical decisions, but still unclear. We conducted a meta-analysis to investigate the

Electronic supplementary material The online version of this article (https://doi.org/10.1007/s12325020-01550-4) contains supplementary material, which is available to authorized users.

L. Wang $\cdot$ X. Guo $\cdot$ X. Xu $\cdot$ X. Qi (ه)

Liver Cirrhosis Study Group, Department of Gastroenterology, General Hospital of Northern Theater Command (Formerly Called General Hospital of Shenyang Military Area), Shenyang, China

e-mail: xingshunqi@126.com

L. Wang

Postgraduate College, Dalian Medical University, Dalian, China

\section{De Stefano}

Dipartimento Di Diagnostica Per Immagini, Radioterapia Oncologica Ed Ematologia, Fondazione Policlinico Universitario Agostino Gemelli IRCCS, Roma, Italy outcomes of anticoagulation for PVT in liver cirrhosis and explore the predictors of portal vein recanalization and bleeding events after anticoagulation.

Methods: All studies regarding anticoagulation for PVT in liver cirrhosis were searched via PubMed, EMBASE, and Cochrane Library databases. Thrombotic outcomes, bleeding events, and survival were compared between anticoagulation and non-anticoagulation groups. Predictors of portal vein recanalization and bleeding events were pooled. Risk ratios (RRs) or mean differences (MDs) with 95\% confidence intervals (CIs) were calculated.

Results: Thirty-three studies including 1696 cirrhotic patients with PVT were included. Anticoagulation significantly increased portal

\author{
A. Plessier \\ Hôpital Beaujon, AP-HP, DHU Unity, Pôle des \\ Maladies de l'Appareil Digestif, Service \\ d'Hépatologie, Centre de Référence Des Maladies \\ Vasculaires du Foie, Inserm U1149, Centre de \\ Recherche Sur L'Inflammation (CRI), Université \\ Paris 7-Denis-Diderot, ERN Rare Liver Clichy, Paris, \\ France \\ C. Noronha Ferreira \\ Serviço de Gastrenterologia E Hepatologia, Hospital \\ de Santa Maria-Centro Hospitalar Universitário \\ Lisboa Norte, Lisboa, Portugal
}


vein recanalization $\quad(\mathrm{RR}=2.61 ; \quad 95 \% \quad \mathrm{CI}$ 1.99-3.43; $P<0.00001)$ and overall survival $(\mathrm{RR}=1.11 ; 95 \%$ CI $1.03-1.21 ; P=0.01)$ and decreased thrombus progression $(\mathrm{RR}=0.26$; 95\% CI 0.14-0.49; $P<0.0001)$. Anticoagulation did not significantly influence overall bleeding $(\mathrm{RR}=0.78 ; 95 \%$ CI $0.47-1.30 ; P=0.34)$. Early initiation of anticoagulation $(\mathrm{RR}=1.58 ; 95 \% \mathrm{CI}$ 1.21-2.07; $P=0.0007)$ significantly increased portal vein recanalization. Child-Pugh class B and $\mathrm{C}(\mathrm{RR}=0.77 ; 95 \%$ CI $0.62-0.95 ; P=0.02)$ and higher MELD score (MD $=-1.48 ; 95 \% \mathrm{CI}$ $-2.20-0.76 ; \quad P<0.0001)$ were significantly associated with decreased portal vein recanalization. No predictor significantly associated with bleeding events was identified.

Conclusions: Early initiation of anticoagulation should be supported in liver cirrhosis with PVT. Predictors of portal vein recanalization should be taken into consideration to identify those who may not benefit from anticoagulation.

Registration: The work was registered in PROSPERO with registration no. CRD42020157142.

Keywords: Anticoagulants; Liver cirrhosis; Meta-analysis; Survival; Venous thrombosis

\section{Key Summary Points}

Why carry out this study?

Benefit and risk of anticoagulation and predictors of portal vein recanalization and bleeding events in cirrhotic patients with portal vein thrombosis remain controversial.

\section{What was learned from the study?}

Anticoagulation can increase the rate of portal vein recanalization, but decrease the rate of thrombus progression in patients with liver cirrhosis and portal vein thrombosis.

Anticoagulation may improve the survival of patients with liver cirrhosis and portal vein thrombosis.
Early initiation of anticoagulation

increases portal vein recanalization, but Child-Pugh class B and C and a higher model for end-stage liver disease score decrease portal vein recanalization.

\section{DIGITAL FEATURES}

This article is published with digital features, including a summary slide, to facilitate understanding of the article. To view digital features for this article go to https://doi.org/10.6084/ m9.figshare.13123214.

\section{INTRODUCTION}

Portal vein thrombosis (PVT) is associated with a variety of disorders, including liver cirrhosis, malignancy, intra-abdominal infection, and abdominal surgery [1]. PVT is commonly observed in liver cirrhosis with a prevalence between 10 and 25\% [2]. PVT can be asymptomatic in most cases, but may be associated with negative outcomes, such as intestinal ischemia [3], severe complications of portal hypertension [4], and increased mortality [5].

The Baveno VI consensus and the American College of Gastroenterology (ACG) clinical guideline recommend anticoagulation primarily for PVT in candidates for liver transplantation (LT) and symptomatic PVT in cirrhotic patients $[6,7]$. By comparison, the indications for anticoagulation are extended to general patients with liver cirrhosis and PVT according to the European Association for the Study of the Liver (EASL) clinical guideline [8]. However, as known, gastroesophageal variceal bleeding (GEVB) is a common manifestation of decompensated cirrhosis. Due to the fear that use of anticoagulants may further increase the risk of bleeding, most clinicians still prefer to postpone or avoid initiating anticoagulant therapy in cirrhotic patients with PVT, especially in those with asymptomatic PVT and non-LT candidates. On the other hand, transient PVT in liver 
cirrhosis that refers to spontaneous resolution of PVT in the absence of any antithrombotic therapy has been increasingly recognized, which further increases the complexity of the decision for anticoagulation in these patients [9].

A meta-analysis by our group in 2015 found that anticoagulation was effective for recanalization of PVT in cirrhotic patients [10]. An updated meta-analysis by Loffredo et al. in 2017 confirmed the effectiveness of anticoagulant therapy over no intervention in recanalizing PVT in cirrhotic patients [11]. Despite these findings, which patients may or may not benefit from anticoagulant therapy is still unclear [12]. Indications for anticoagulant therapy in patients with liver cirrhosis who develop PVT need to be clarified. Notably, there has been a remarkable increase in the number of recently published studies involving anticoagulation for PVT in liver cirrhosis since the updated metaanalysis by Loffredo et al. was published. Thus, it would be useful to perform a critical analysis on this topic.

This systematic review and meta-analysis has four major objectives. The first is to report the rates of portal vein recanalization and bleeding in cirrhotic patients with PVT receiving anticoagulation. The second is to compare the rates of portal vein recanalization, bleeding, and survival between cirrhotic patients with PVT who received and did not receive anticoagulation. The third is to analyze the characteristics of cirrhotic patients with PVT who obtained portal vein recanalization from anticoagulation. The fourth is to explore the predictors of portal vein recanalization and bleeding events after anticoagulation.

\section{METHODS}

The systematic review and meta-analysis was conducted according to the PRISMA checklist as shown in Supplementary Table 1.

This article is based on previously published studies and does not involve any new studies of human or animal subjects performed by any of the authors.

\section{Registration}

The work was registered in PROSPERO with registration no. CRD42020157142.

\section{Literature Source}

All published papers were searched via the PubMed, EMBASE, and Cochrane Library databases. The last search was conducted on March 4, 2020 .

\section{Search Strategy}

The search items are shown in Appendix.

\section{Eligibility Criteria}

All clinical studies that assessed the effect and/or bleeding risk of anticoagulation for PVT in cirrhotic patients and/or the predictors of portal vein recanalization and/or bleeding events after anticoagulation for PVT were included. Publication language, date, and status were not limited.

\section{Exclusion Criteria}

Exclusion criteria were as follows: (1) duplicates; (2) case reports, letters, comments, and/or editorials; (3) reviews and/or meta-analyses; (4) guidelines, consensus, or reports; (5) experimental or animal studies; (6) irrelevant studies that did not evaluate the efficacy or safety of anticoagulation in cirrhotic patients with PVT; (7) studies in which only non-cirrhotic PVT or malignant PVT was included; (8) studies in which PVT developed after splenectomy, splenic arterial embolization, transjugular intrahepatic portosystemic shunt (TIPS), LT, or other major surgical interventions; (9) studies in which anticoagulation was not given; (10) studies in which anticoagulation was given for 
the prevention of PVT; (11) studies in which detailed data were lacking; (12) studies in which fewer than ten cirrhotic patients with PVT receiving anticoagulation were enrolled.

\section{Data Extraction}

Characteristics of included studies and patients were extracted, including first author, publication year, region, enrollment period, study design, study population, features of PVT, exclusion of hepatocellular carcinoma (HCC), exclusion of cavernous transformation of portal vein (CTPV), exclusion of isolated thrombosis within the superior mesenteric vein (SMV) or splenic vein (SV), exclusion of patients receiving antiplatelet treatment, exclusion of patients receiving thrombolytic treatment, interval between diagnosis of PVT and initiation of anticoagulation, baseline gastroesophageal varices, follow-up duration, and type and dose of anticoagulants. Rates of portal vein recanalization, thrombus progression, re-thrombosis, bleeding events, including overall bleeding, major bleeding, upper gastrointestinal bleeding (UGIB), GEVB, and deaths due to bleeding events, and survival in anticoagulation and non-anticoagulation groups were also extracted. In addition, the characteristics of cirrhotic patients with PVT who received anticoagulation were reviewed, and the predictors of portal vein recanalization and bleeding events after anticoagulation were extracted.

\section{Definitions}

Overall recanalization included complete and partial recanalization. Complete recanalization was defined as complete resolution of previously detected thrombus. Because the definition of partial recanalization was very inconsistent among the included studies or unclear in some studies, this outcome was not evaluated in the present work.

Thrombus progression was considered if there was a significant increase in thrombus lumen occupancy or thrombus extension to unaffected segments of the splenoportomesenteric axis.
Major bleeding was defined as fatal bleeding, symptomatic bleeding in a critical location or organ, a fall in hemoglobin level $\geq 2 \mathrm{~g} / \mathrm{dl}$, or a requirement of red blood cell transfusion $\geq 2$ units.

UGIB mainly included bleeding caused by ulcers, GEVB, and portal hypertensive gastropathy.

\section{Study Quality}

The study quality was assessed by the Newcastle-Ottawa Scale (NOS) criteria for cohort studies [13]. Three major parts are assessed: (1) selection (score 0-4), (2) comparability (score $0-2$ ), and (3) outcome (score 0-3). The maximum score is 9 . A score of $0-3,4-6$, and 7-9 represents low, moderate, and high quality, respectively.

\section{Statistical Analysis}

We performed the meta-analyses by using random-effect models in Review Manager 5.3 (Cochrane Collaboration, Nordic Cochrane Centre, Copenhagen), Stats Direct 2.8.0 (StatsDirect Ltd., Sale, Cheshire, UK), and STATA 12.0 (Stata Corp, College Station, TX). Pooled proportions and risk ratios (RRs) with 95\% confidence intervals (CIs) were calculated for categorical variables. Mean differences (MDs) with $95 \%$ CIs were calculated for continuous variables. Cochrane $\mathrm{Q}$ test and the $I^{2}$ statistics were employed to assess the heterogeneity, and $P<0.1$ or $I^{2}>50 \%$ was considered as a statistically significant heterogeneity. Meta-regression and subgroup analyses were employed to explore the sources of heterogeneity. In the meta-regression analyses, publication year, region, study design, study quality, exclusion of HCC, exclusion of CTPV, exclusion of isolated thrombosis within SMV or SV, exclusion of patients receiving antiplatelet treatment, exclusion of patients receiving thrombolytic treatment, and type of anticoagulants were used as covariates. Subgroup analyses were also performed in terms of the variables mentioned above. Sensitivity analyses were performed to assess the impact of each individual study on 
the stability of statistical results by removing studies one by one from a meta-analysis. Publication bias was evaluated with the Egger test, and $P<0.1$ was considered as a statistically significant publication bias. We also drew the scattered plots and performed the Spearman correlation analysis in the IBM SPSS 26.0 (IBM Corp., Armonk, NY, USA) to explore the correlation between patient characteristics and portal vein recanalization rate after anticoagulation. Coefficients, which are denoted as $r_{\mathrm{s}}$, were calculated. A two-sided $P<0.05$ indicates a statistical significance.

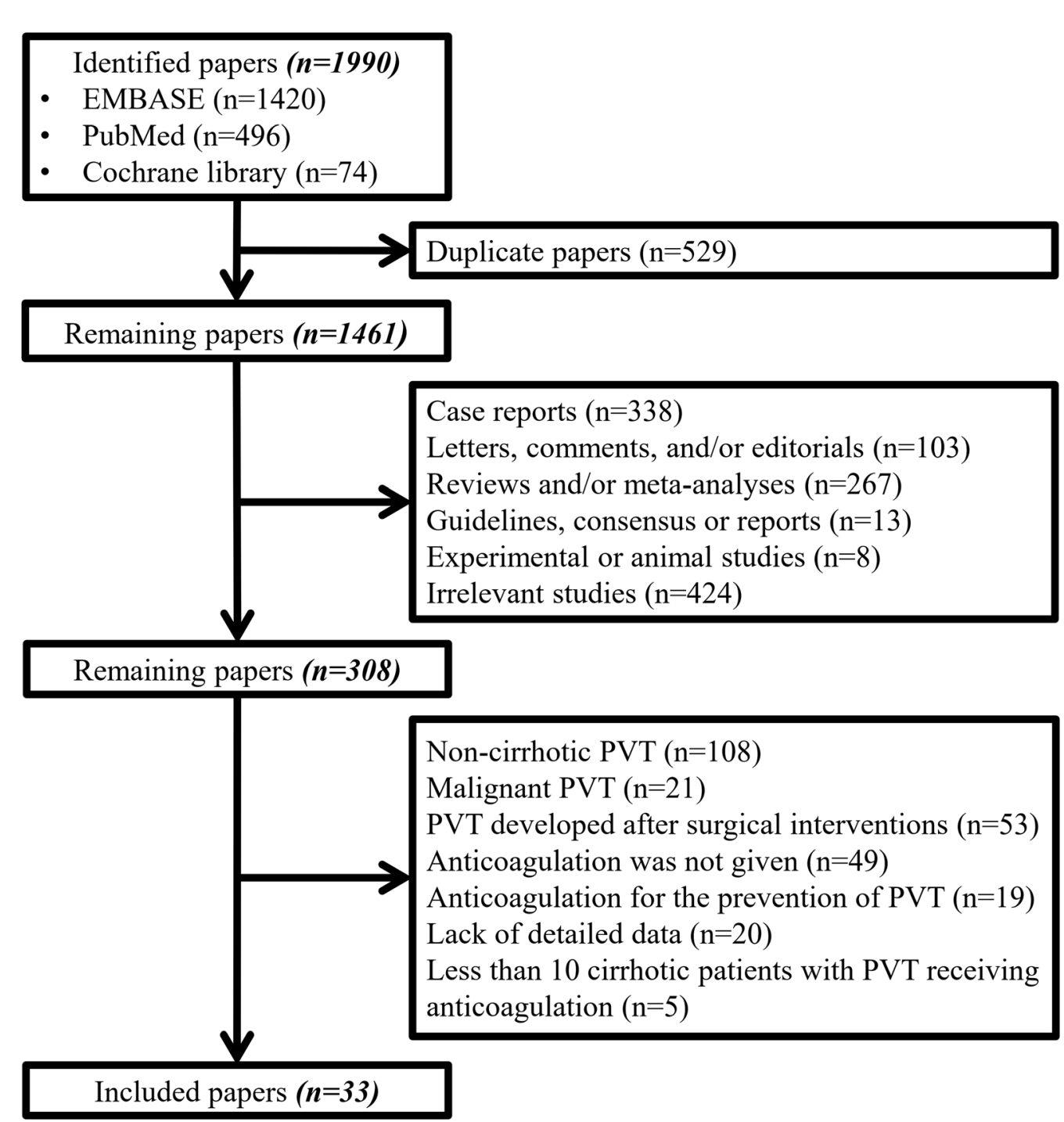

\section{RESULTS}

\section{Included Studies}

A total of 33 studies including 1696 patients with liver cirrhosis and PVT were eligible (Fig. 1). Notably, six studies evaluated the efficacy of anticoagulant therapy for PVT in liver cirrhosis, but they were finally excluded. This was mainly because they included patients undergoing splenectomy [14-16], splenic arterial embolization [17], or TIPS [18, 19].

Main characteristics of studies are summarized in Table 1 . The sample size ranged from 16

Fig. 1 Flowchart of study selection 


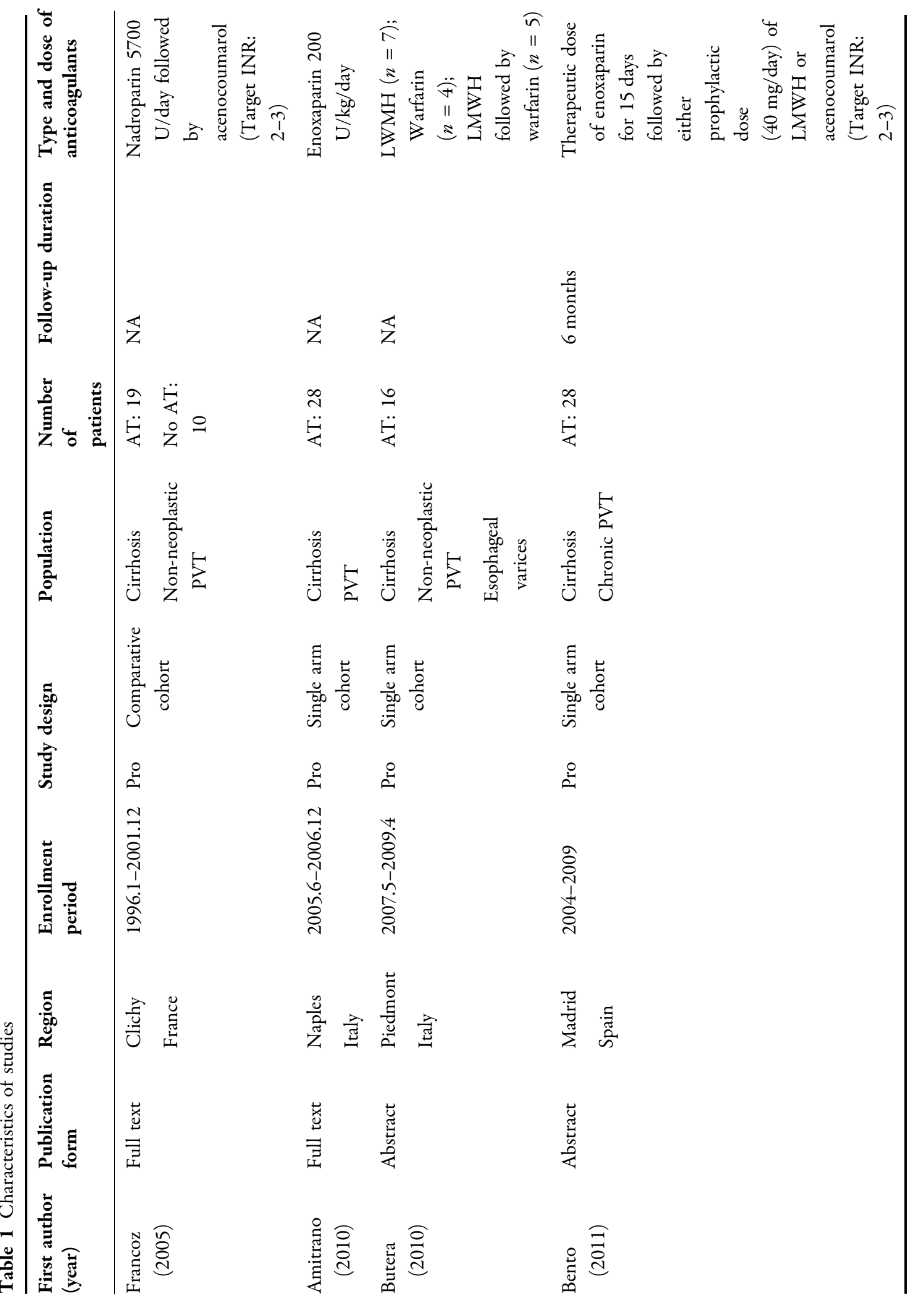




\begin{tabular}{|c|c|c|c|}
\hline 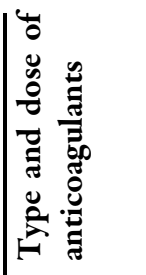 & 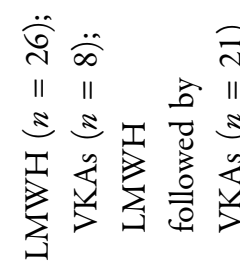 & 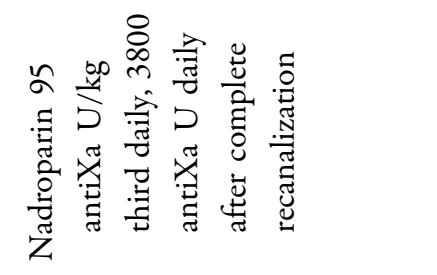 & $\sum_{\substack{3\\
}}^{I}$ \\
\hline & 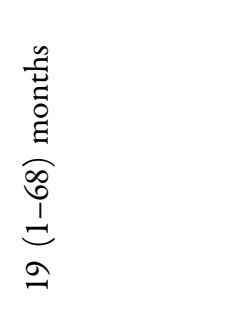 & 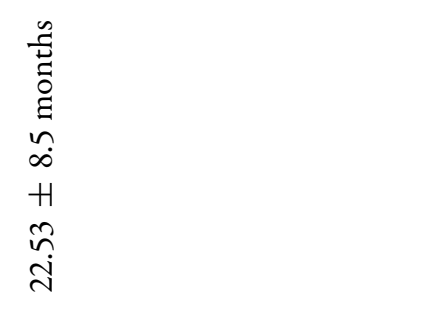 & 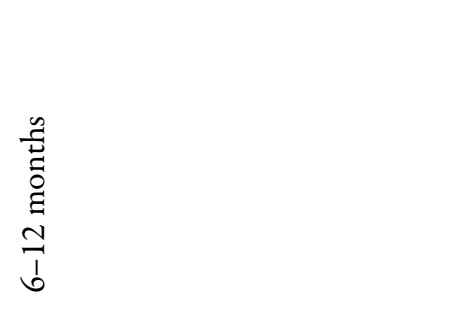 \\
\hline 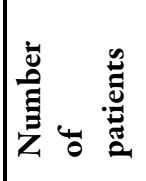 & $\begin{array}{l}\tilde{n} \\
\ddot{*}\end{array}$ & 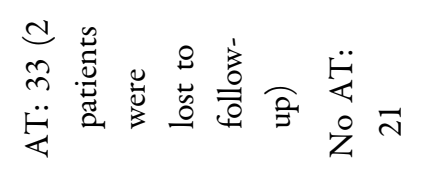 & $\begin{array}{ll}\simeq & \ddot{U} \\
\ddot{H} & \stackrel{0}{Z}\end{array}$ \\
\hline & 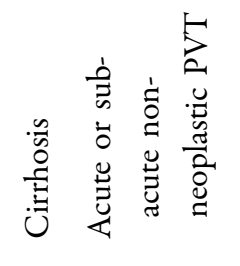 & 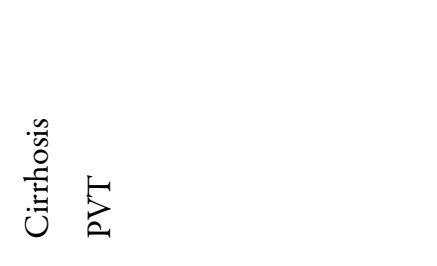 & 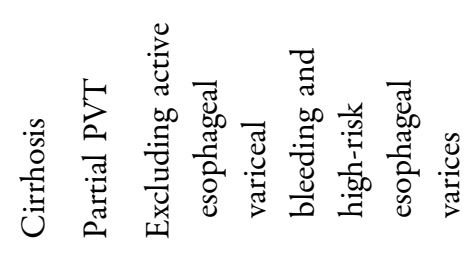 \\
\hline & 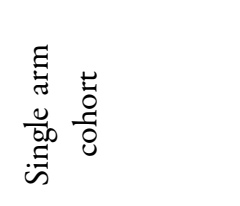 & 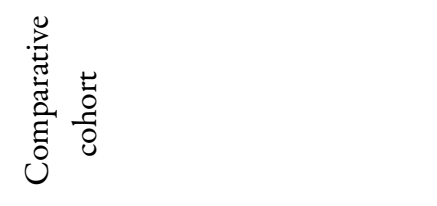 & 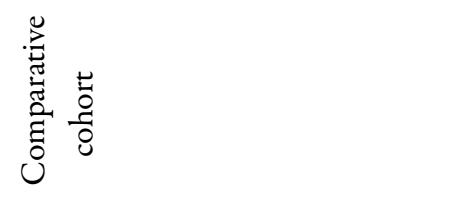 \\
\hline & $\stackrel{2}{2}$ & $\stackrel{2}{2}$ & : \\
\hline 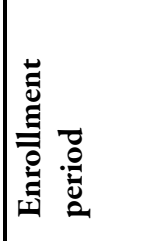 & 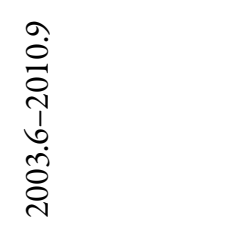 & 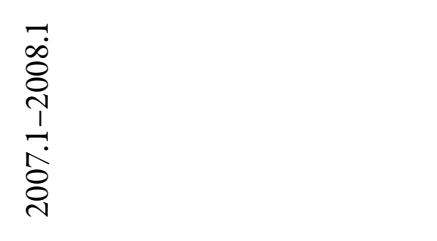 & $\overleftrightarrow{Z}$ \\
\hline & 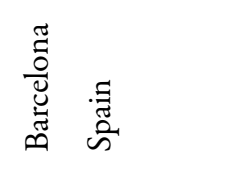 & 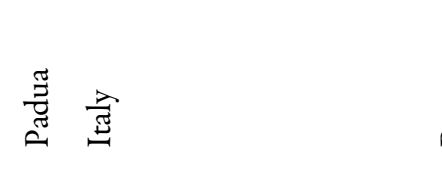 & 苛 䲩 \\
\hline 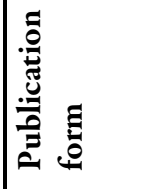 & 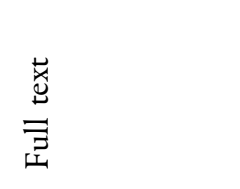 & 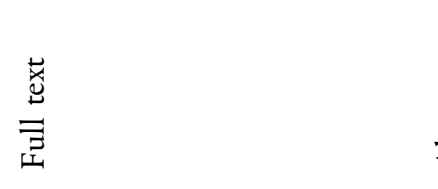 & 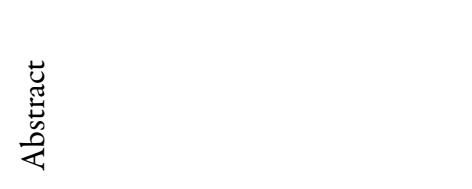 \\
\hline 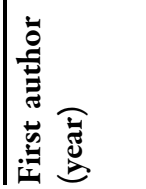 & 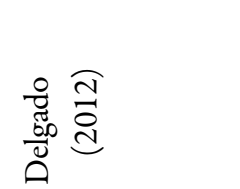 & 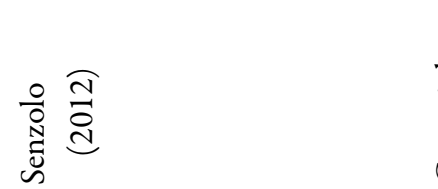 & 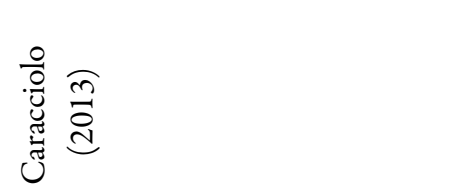 \\
\hline
\end{tabular}




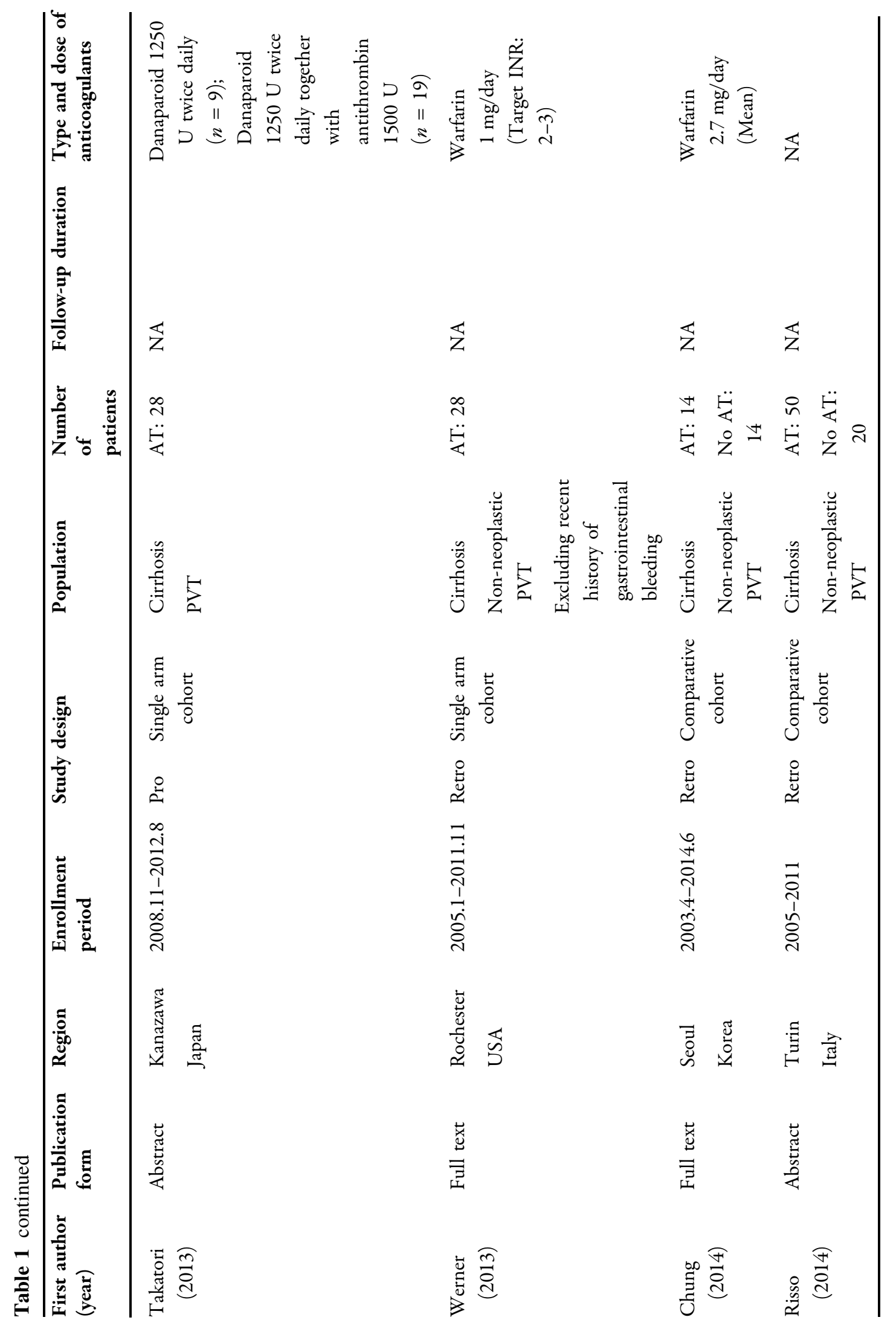




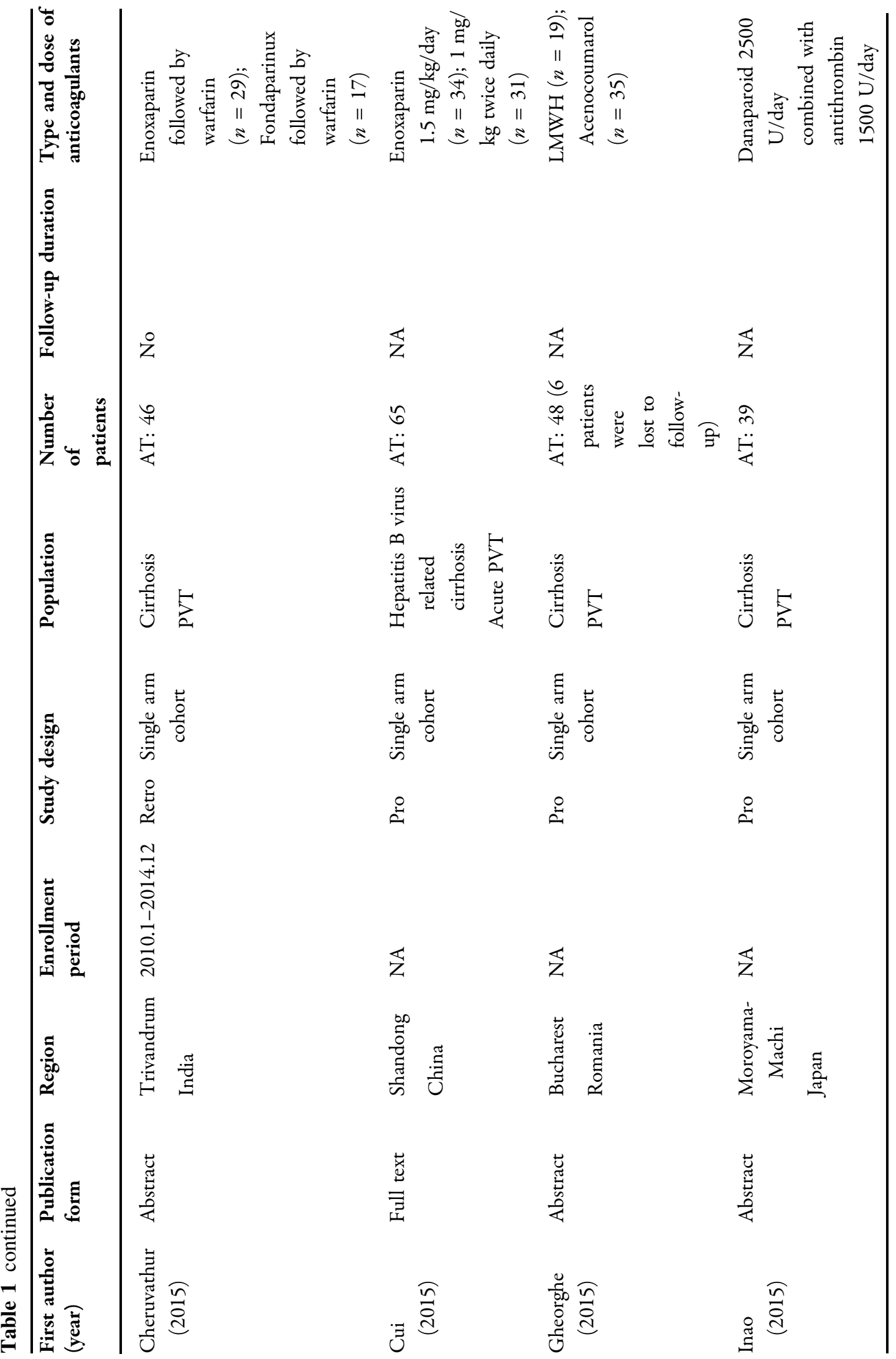




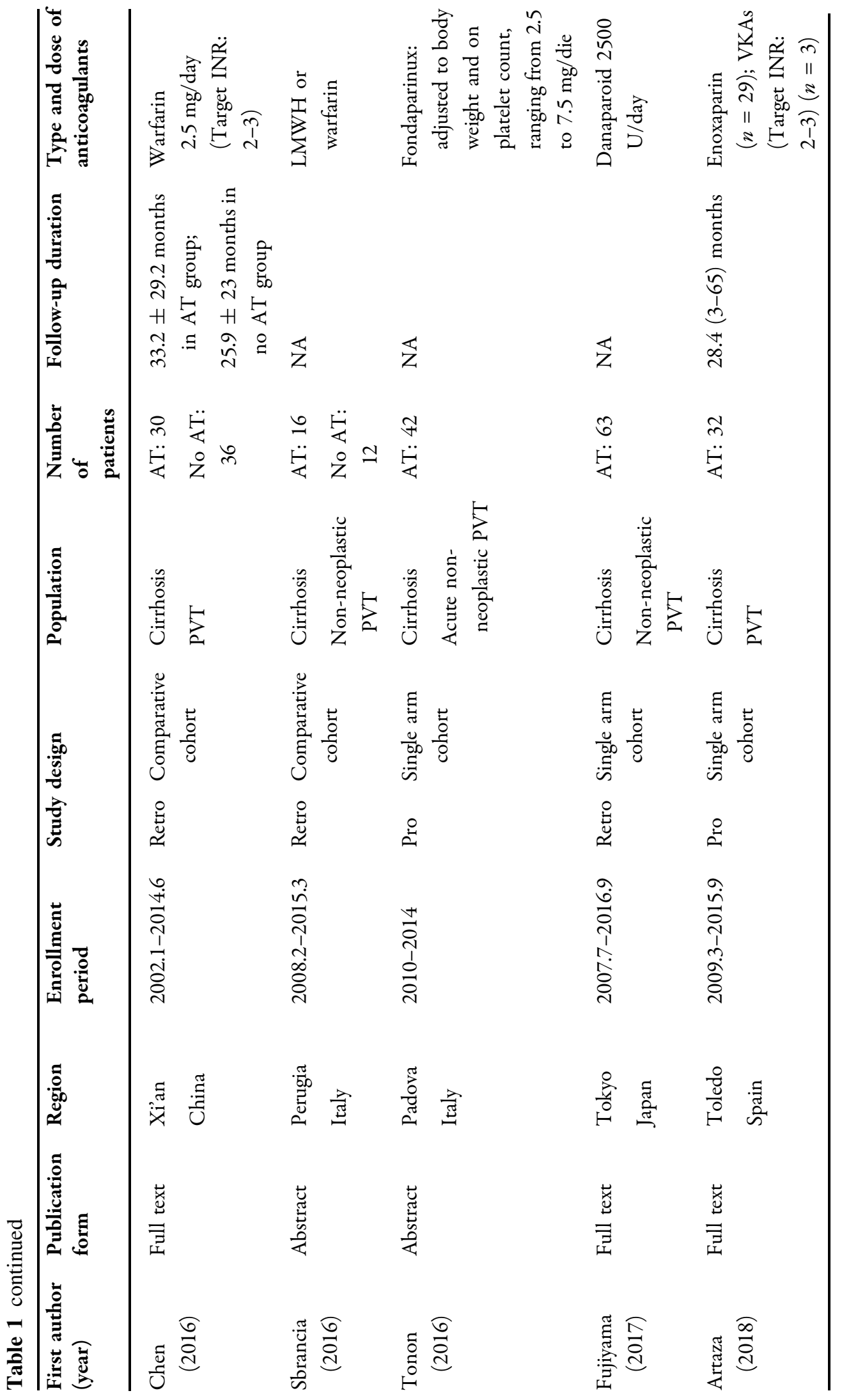




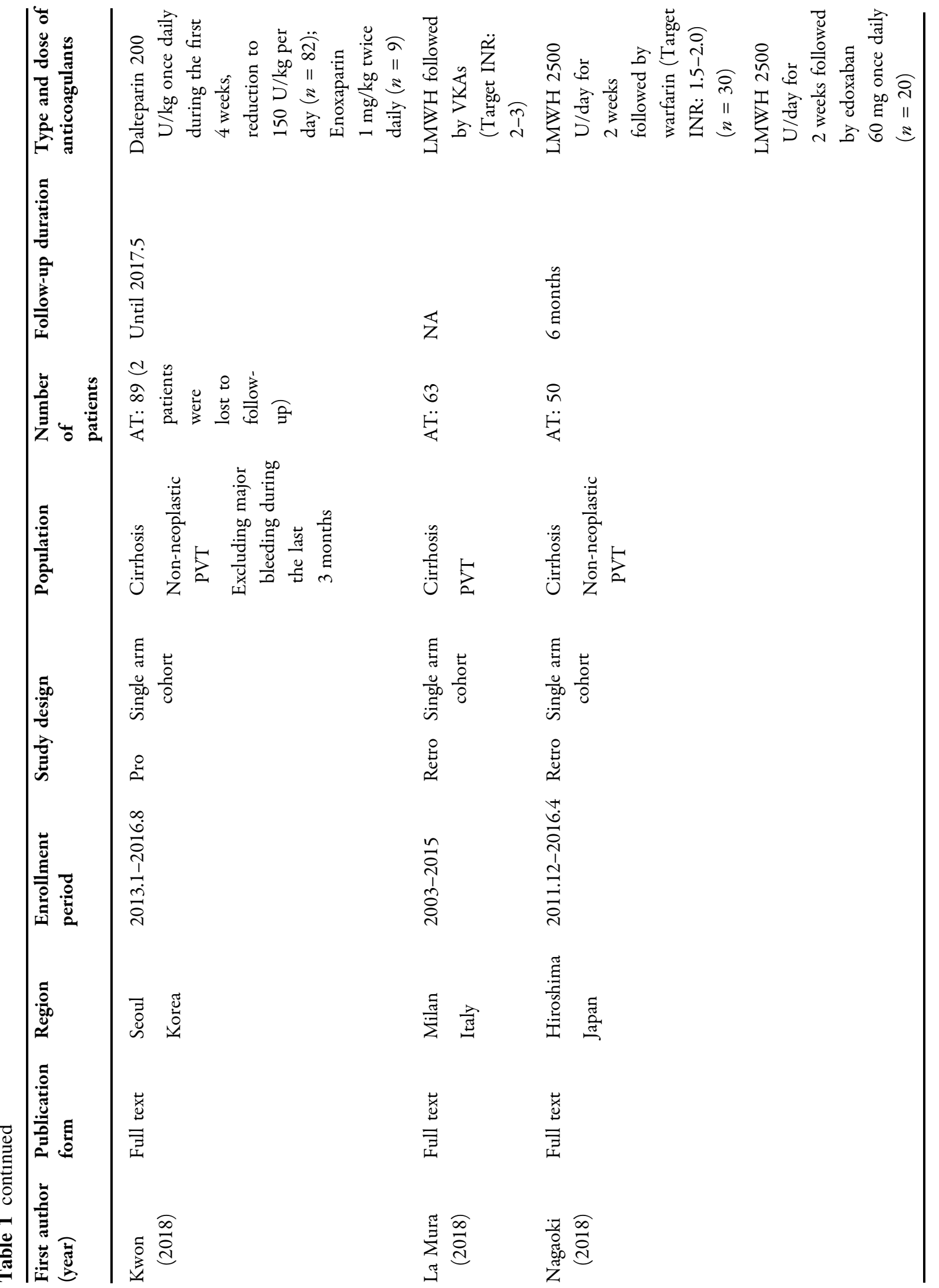




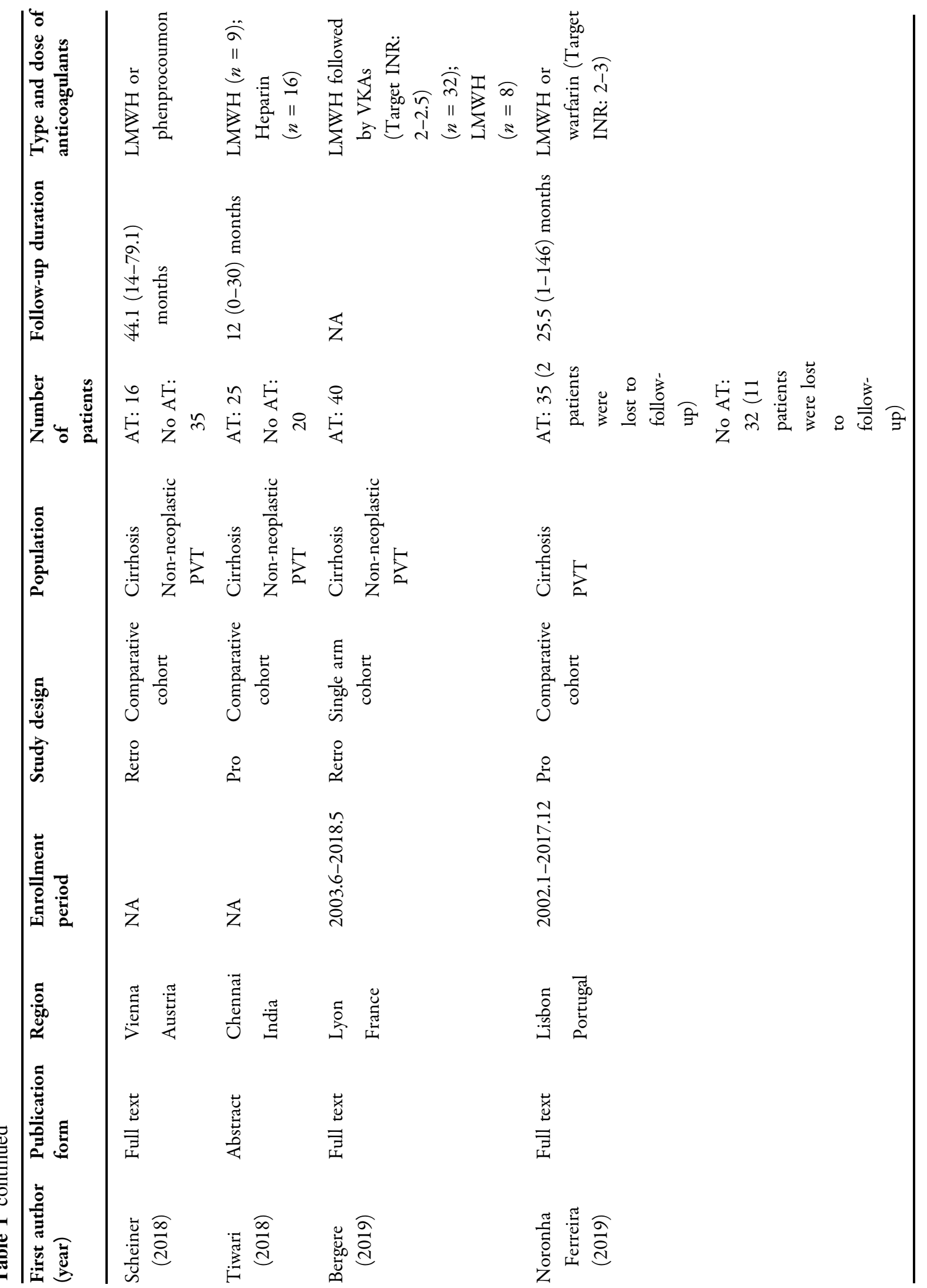




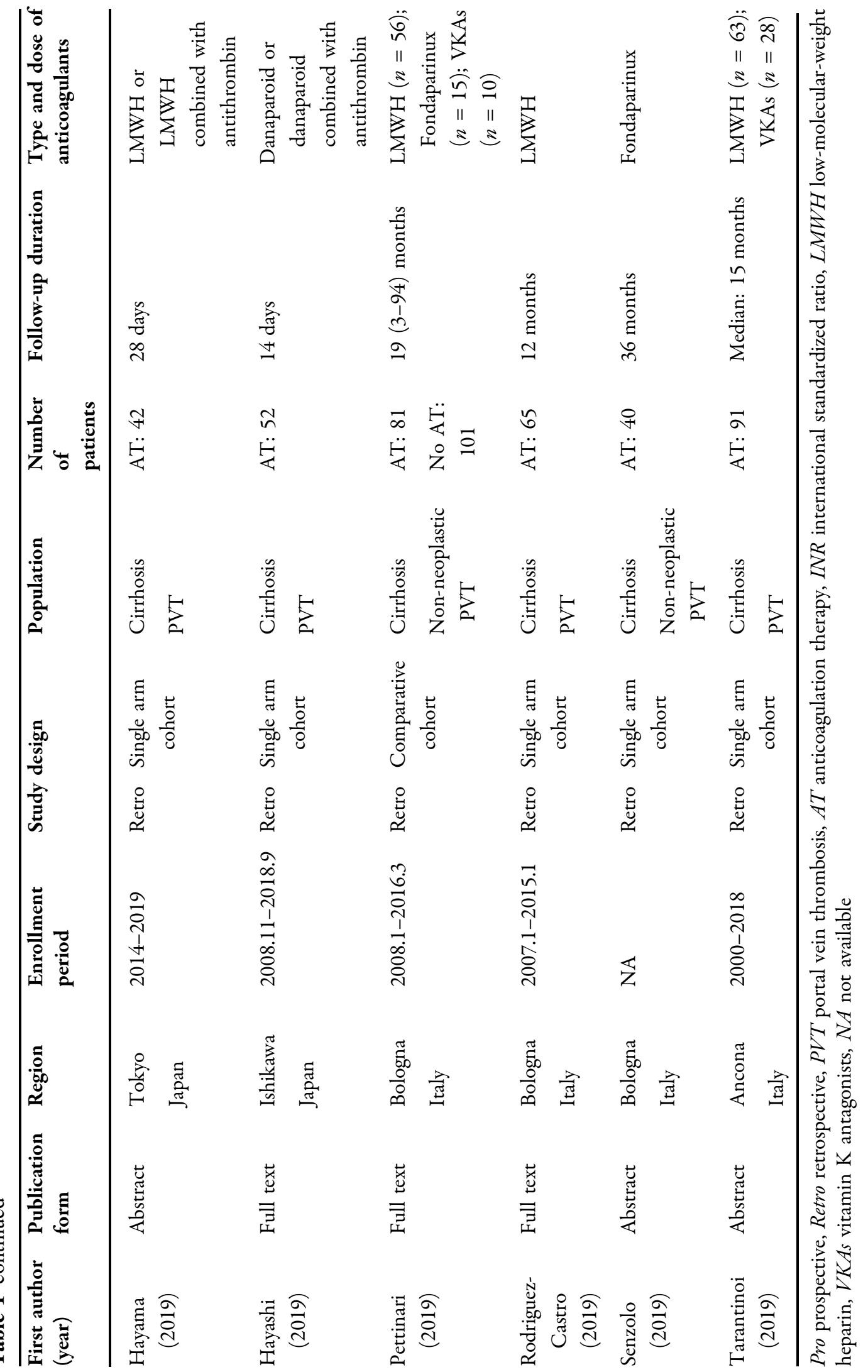


to 182 . Nineteen studies were published as full texts [14, 20-37] and 14 as abstracts [38-51]. They were published between 2005 and 2019 . Twenty of them were performed in Europe [21-25, 30, 32-36, 38-40, 42, 44, 46, 47, 49, 50]. Fifteen studies were of prospective nature [22-25, 28, 30, 31, 36, 38, 39, 41, 44, 45, 47, 48]. Eleven studies were comparative cohort studies with a control group in which anticoagulation was not given $[14,21,22,25,27,34,36,40,42,46,48]$. As for the type of anticoagulants, low-molecularweight heparin (LMWH) alone was given in six studies [23, 28, 29, 31, 35, 40], vitamin $\mathrm{K}$ antagonists (VKAs) alone in three studies $[14,26,27]$, fondaparinux alone in two studies [47, 49], direct-acting oral anticoagulants (DOACs) alone in one study [20], LMWH followed by VKAs in three studies [20, 22, 32], and LMWH in combination with antithrombin in one study [45].

Main characteristics of patients are summarized in Supplementary Table 2. Ten studies clearly excluded patients with HCC $[14,21,23,25,28,30,32,35,38,40]$, nine excluded patients with CTPV $[14,23,24,26,28,30,33,40,47]$, five excluded patients with isolated thrombosis within SMV or SV $[14,25,30,35,36]$, three excluded patients receiving antiplatelet treatment $[25,28,35]$, and one excluded patients receiving thrombolytic treatment [14].

\section{Study Quality}

The NOS score ranged from 4 to 9 points. Twenty-six studies were considered to be of moderate quality, and seven were of high quality $[14,21,25,27,36,40,48]$ (Supplementary Table 3).

\section{Overall Portal Vein Recanalization}

Twenty-nine studies reported the rate of overall portal vein recanalization after anticoagulation, and the pooled rate was $71.5 \%$ (95\% CI 66.0-76.7\%) (Supplementary Fig. 1). There was a statistically significant heterogeneity $\left(I^{2}=77.4 \% ; \quad P<0.0001\right) . \quad$ Meta-regression analysis found that the heterogeneity might be related to the publication year (Supplementary Table 4). In subgroup analyses of studies excluding HCC, excluding isolated thrombosis within SMV or SV, excluding patients receiving antiplatelet treatment, using LMWH alone, using VKAs alone, and using fondaparinux alone, the heterogeneity became not statistically significant (Supplementary Table 5). In sensitivity analyses, the pooled rates of overall portal vein recanalization were comparable after eliminating individual studies one by one, and the heterogeneity remained statistically significant (Supplementary Table 6).

Seven studies compared the rate of overall portal vein recanalization between patients who received and did not receive anticoagulation. Meta-analysis demonstrated that anticoagulation significantly increased the rate of overall portal vein recanalization $(\mathrm{RR}=2.61 ; 95 \% \mathrm{CI}$ $1.99-3.43 ; P<0.00001)$. There was no statistically significant heterogeneity among studies $\left(I^{2}=0 \% ; P=0.66\right)$ (Fig. 2a).

\section{Complete Portal Vein Recanalization}

Twenty-seven studies reported the rate of complete portal vein recanalization after anticoagulation, and the pooled rate was $40.8 \%$ (95\% CI $35.2-46.5 \%)$. There was a statistically significant heterogeneity $\left(I^{2}=75.1 \% ; P<0.0001\right)$. Metaregression analysis found that the heterogeneity might be related to the exclusion of CTPV (Supplementary Table 4). In subgroup analyses of studies performed in Asia and studies using VKAs alone, the heterogeneity became not statistically significant (Supplementary Table 5). In sensitivity analyses, the pooled rates of complete portal vein recanalization were comparable after eliminating individual studies one by one, and the heterogeneity remained statistically significant (Supplementary Table 6).

Six studies compared the rate of complete portal vein recanalization between patients who received and did not receive anticoagulation. Meta-analysis demonstrated that anticoagulation significantly increased the rate of complete portal vein recanalization $(\mathrm{RR}=2.14 ; 95 \% \mathrm{CI}$ $1.30-3.50 ; P=0.003)$. There was no statistically 


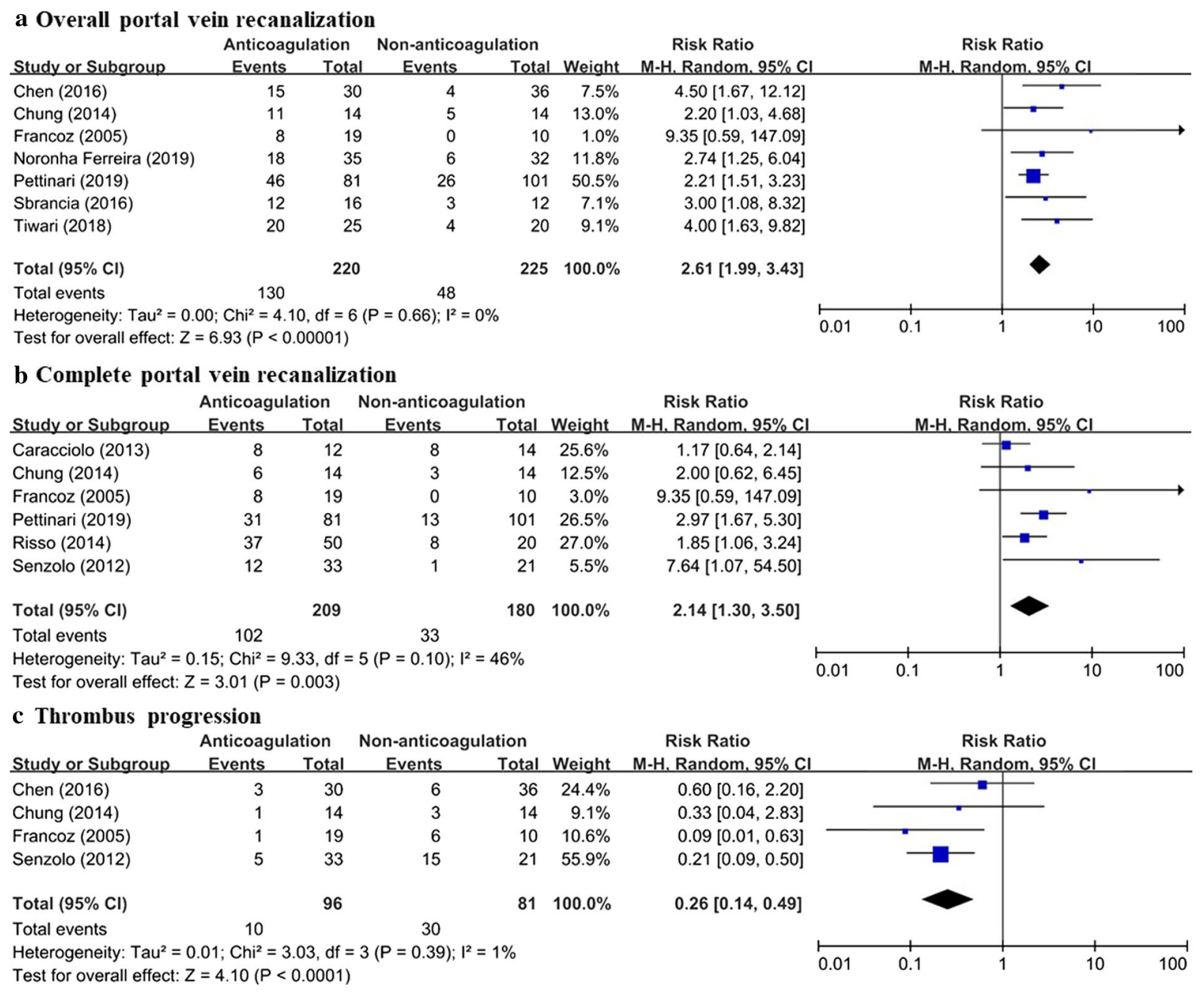

Fig. 2 Comparison of portal vein recanalization and thrombus progression between anticoagulation and nonanticoagulation groups

significant heterogeneity among studies $\left(I^{2}=46 \% ; P=0.10\right)$ (Fig. $\left.2 \mathrm{~b}\right)$.

\section{Thrombus Progression}

Twenty-three studies reported the rate of thrombus progression after anticoagulation, and the pooled rate was $6.9 \% \quad(95 \%$ CI $3.1-12 \%)$. There was a statistically significant heterogeneity $\left(I^{2}=84.9 \% ; P<0.0001\right)$. Metaregression analysis did not find any source of heterogeneity (Supplementary Table 4). In subgroup analyses of comparative cohort studies and high-quality studies, the heterogeneity became not statistically significant
(Supplementary Table 7). In sensitivity analyses, the pooled rates of thrombus progression were comparable after eliminating individual studies one by one, and the heterogeneity remained statistically significant (Supplementary Table 6).

Four studies compared the rate of thrombus progression between patients who received and did not receive anticoagulation. Meta-analysis demonstrated that anticoagulation significantly decreased the rate of thrombus progression $(\mathrm{RR}=0.26 ; \quad 95 \%$ CI $0.14-0.49 ; \quad P<0.0001)$. There was no statistically significant heterogeneity among studies ( $\left.I^{2}=1 \% ; \quad P=0.39\right)$ (Fig. 2c). 
Table 2 Predictors of portal vein recanalization and bleeding events: results of meta-analyses

\begin{tabular}{|c|c|c|c|c|c|}
\hline \multirow[t]{2}{*}{ Predictors } & \multirow{2}{*}{$\begin{array}{l}\text { No. } \\
\text { studies }\end{array}$} & \multirow[t]{2}{*}{ Effect size $(95 \% \mathrm{CI})$} & \multirow[t]{2}{*}{$P$ value } & \multicolumn{2}{|c|}{ Heterogeneity } \\
\hline & & & & $I^{2}$ & $P$ value \\
\hline \multicolumn{6}{|c|}{ Predictors of portal vein recanalization: results of meta-analyses } \\
\hline Child-Pugh class (B and $\mathrm{C}$ vs. A) & 3 & $\mathrm{RR}=0.77(0.62,0.95)$ & 0.020 & $0 \%$ & 0.66 \\
\hline $\begin{array}{l}\text { Interval between PVT diagnosis and } \\
\text { initiation of anticoagulation (early vs. late) }\end{array}$ & 6 & $\mathrm{RR}=1.58(1.21,2.07)$ & 0.0007 & $30 \%$ & 0.21 \\
\hline$\leq 14$ days vs. $>14$ days & 1 & $\mathrm{RR}=1.79(1.00,3.18)$ & 0.050 & - & - \\
\hline$\leq 1$ month vs. $>1$ month & 1 & $\mathrm{RR}=1.13(0.73,1.72)$ & 0.590 & - & - \\
\hline$\leq 3$ months vs. $>3$ months & 1 & $\mathrm{RR}=1.49(1.10,2.02)$ & 0.010 & - & - \\
\hline$\leq 6$ months vs. $>6$ months & 3 & $\mathrm{RR}=2.11(1.29,3.45)$ & 0.003 & $10 \%$ & 0.33 \\
\hline Duration of anticoagulation ( $\leq 6$ months) & 2 & $\mathrm{RR}=1.53(1.09,2.16)$ & 0.010 & $0 \%$ & 0.35 \\
\hline Gender (male) & 8 & $\mathrm{RR}=1.14(0.91,1.43)$ & 0.250 & $54 \%$ & 0.03 \\
\hline Previous portal hypertensive bleeding & 2 & $\mathrm{RR}=0.66(0.42,1.04)$ & 0.070 & $57 \%$ & 0.13 \\
\hline Ascites & 4 & $\mathrm{RR}=1.01(0.78,1.30)$ & 0.950 & $47 \%$ & 0.13 \\
\hline Hepatic encephalopathy & 3 & $\mathrm{RR}=0.92(0.68,1.25)$ & 0.600 & $0 \%$ & 0.96 \\
\hline Hepatocellular carcinoma & 3 & $\mathrm{RR}=0.89(0.70,1.13)$ & 0.340 & $0 \%$ & 0.97 \\
\hline Thrombophilia & 2 & $\mathrm{RR}=0.77(0.16,3.72)$ & 0.750 & $85 \%$ & 0.01 \\
\hline Cavernous transformation of portal vein & 2 & $\mathrm{RR}=0.49(0.21,1.15)$ & 0.100 & $0 \%$ & 0.56 \\
\hline Isolated splenic vein thrombosis & 2 & $\mathrm{RR}=1.27(0.90,1.78)$ & 0.170 & $0 \%$ & 0.68 \\
\hline $\begin{array}{l}\text { Isolated superior mesenteric } \\
\text { vein thrombosis }\end{array}$ & 2 & $\mathrm{RR}=0.92(0.68,1.25)$ & 0.600 & $0 \%$ & 0.32 \\
\hline Complete PVT & 3 & $\mathrm{RR}=0.80(0.59,1.09)$ & 0.150 & $0 \%$ & 0.99 \\
\hline Age (years) & 5 & $\mathrm{MD}=1.84(-1.96,5.63)$ & 0.340 & $29 \%$ & 0.23 \\
\hline Child-Pugh Score & 2 & $\mathrm{MD}=-0.44(-2.29,1.41)$ & 0.640 & $73 \%$ & 0.05 \\
\hline MELD score & 3 & $\mathrm{MD}=-1.48(-2.20,-0.76)$ & $<0.0001$ & $0 \%$ & 0.64 \\
\hline Platelet count $\left(10^{9} / 1\right)$ & 5 & $\mathrm{MD}=7.24(-2.52,17.00)$ & 0.150 & $54 \%$ & 0.07 \\
\hline Serum creatinine $(\mathrm{mg} / \mathrm{dl})$ & 3 & $\mathrm{MD}=0.06(0.00,0.12)$ & 0.050 & $8 \%$ & 0.34 \\
\hline International normalized ratio & 4 & $\mathrm{MD}=-0.04(-0.08,0.00)$ & 0.080 & $42 \%$ & 0.16 \\
\hline \multicolumn{6}{|c|}{ Predictors of bleeding events: results of meta-analyses } \\
\hline Type of anticoagulants (LMWH vs. VKAs) & 2 & $R R=2.30(0.20,26.43)$ & 0.510 & $65 \%$ & 0.51 \\
\hline Child-Pugh Score & 2 & $\mathrm{MD}=-0.22(-1.15,0.72)$ & 0.650 & $0 \%$ & 0.42 \\
\hline MELD score & 2 & $\mathrm{MD}=-0.19(-1.5,1.12)$ & 0.780 & $0 \%$ & 0.14 \\
\hline Platelet count $\left(10^{9} / 1\right)$ & 2 & $\mathrm{MD}=-33.71(-98.18,30.76)$ & 0.310 & $85 \%$ & 0.01 \\
\hline
\end{tabular}


Table 2 continued

\begin{tabular}{llllll}
\hline Predictors & $\begin{array}{l}\text { No. } \\
\text { studies }\end{array}$ & Effect size (95\% CI) & $\boldsymbol{P}$ value & \multicolumn{2}{c}{ Heterogeneity } \\
\hline Duration of anticoagulation (months) & 2 & $\mathrm{MD}=-0.40(-15.21,14.41)$ & 0.960 & $54 \%$ & 0.14 \\
\hline
\end{tabular}

Bolditalics indicate $P$ value $<0.05$

$R R$ risk ratio, $M D$ mean difference, $C I$ confidence interval, $P V T$ portal vein thrombosis, $M E L D$ model for end-stage liver disease, $L M W H$ low-molecular-weight heparin, $V K A s$ vitamin $\mathrm{K}$ antagonists

\section{Re-Thrombosis}

Nine studies reported the rate of re-thrombosis after stopping anticoagulation, and the pooled rate was $46.7 \%$ (95\% CI 37.7-69.3\%). There was no statistically significant heterogeneity $\left(I^{2}=36 \% ; P=0.1306\right)$.

No data reported the rate of re-thrombosis between patients who stopped and did not stop anticoagulation.

\section{Overall Bleeding}

Twenty-four studies reported the rate of overall bleeding after anticoagulation, and the pooled rate was $10.3 \%$ (95\% CI 6.4-15.0\%). There was a statistically significant heterogeneity $\left(I^{2}=79.8 \% ; P<0.0001\right)$. Meta-regression analysis found that the heterogeneity might be related to the publication year (Supplementary Table 4). In subgroup analyses of studies excluding patients receiving antiplatelet treatment, the heterogeneity became not statistically significant (Supplementary Table 7). In sensitivity analyses, the pooled rates of overall bleeding were comparable after eliminating individual studies one by one, and the heterogeneity remained statistically significant (Supplementary Table 6).

Four studies compared the rate of overall bleeding between patients who received and did not receive anticoagulation. Meta-analysis demonstrated that anticoagulation did not significantly increase the rate of overall bleeding $(\mathrm{RR}=0.78 ; 95 \%$ CI $0.47-1.30 ; P=0.34)$. There was no statistically significant heterogeneity among studies $\left(I^{2}=0 \% ; P=0.45\right)$ (Fig. 3a).

\section{Major Bleeding}

Nineteen studies reported the rate of major bleeding after anticoagulation, and the pooled rate was $2.8 \%$ (95\% CI $1.4-4.6 \%)$. There was a statistically significant heterogeneity $\left(I^{2}=43.3 \%\right.$; $\left.P=0.0235\right)$. Meta-regression analysis did not find any source of heterogeneity (Supplementary Table 4). In subgroup analyses of prospective studies, comparative cohort studies, high-quality studies, and studies published before 2015, performed in Asia, excluding CTPV, using LMWH alone, using VKAs alone, using fondaparinux alone, and using LMWH followed by VKAs, the heterogeneity became not statistically significant (Supplementary Table 8). Sensitivity analyses suggested that the heterogeneity might be attributed to the study by La Mura (2018) (Supplementary Table 6).

No data reported the rate of major bleeding between patients who received and did not receive anticoagulation.

\section{UGIB}

Eighteen studies reported the rate of UGIB after anticoagulation, and the pooled rate was 3.2\% (95\% CI $1.7-5.1 \%)$. There was a statistically significant heterogeneity $\left(I^{2}=38.0 \%\right.$; $P=0.0524)$. Meta-regression analysis did not find any source of heterogeneity (Supplementary Table 4). In subgroup analyses of prospective studies, comparative cohort studies, highquality studies, and studies published before 2015, performed in Asia, performed in Europe, excluding HCC, not excluding CTPV, excluding 


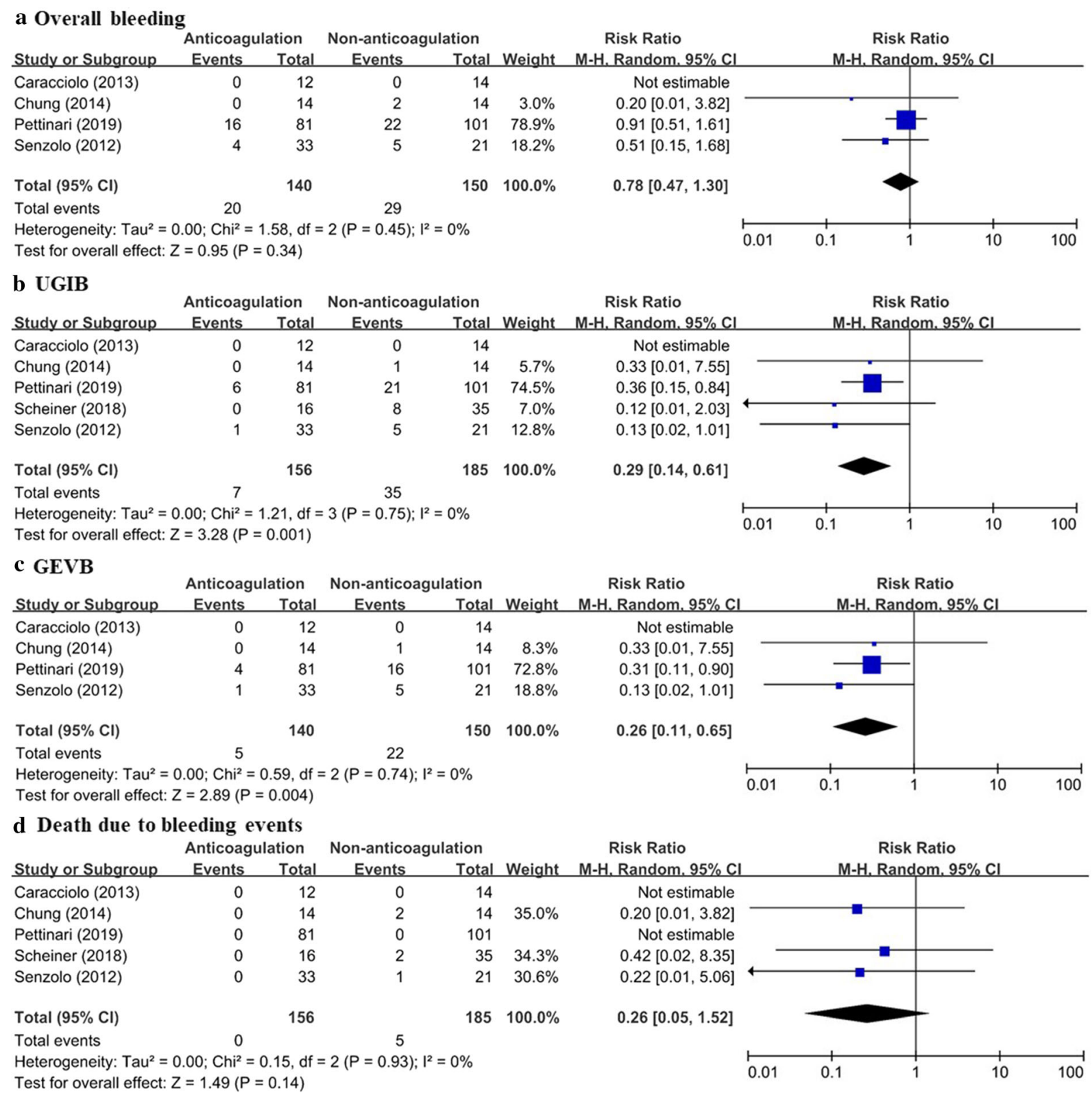

Fig. 3 Comparison of overall bleeding, UGIB, GEVB, and death due to bleeding events between anticoagulation and nonanticoagulation groups

isolated thrombosis within SMV or SV, excluding patients receiving antiplatelet treatment, using LMWH alone, using VKAs alone, and using LMWH followed by VKAs, the heterogeneity became not statistically significant (Supplementary Table 8). Sensitivity analyses suggested that the heterogeneity might be attributed to the studies by Cui (2015),
Fujiyama (2017), Bergere (2019), or Pettinari (2019) (Supplementary Table 6).

Five studies compared the rate of UGIB between patients who received and did not receive anticoagulation. Meta-analysis demonstrated that anticoagulation significantly decreased the rate of UGIB (RR $=0.29 ; 95 \%$ CI $0.14-0.61 ; P=0.001)$. There was no statistically 
significant heterogeneity $\left(I^{2}=0 \% ; P=0.75\right)$ (Fig. 3b).

\section{GEVB}

Seventeen studies reported the rate of GEVB after anticoagulation, and the pooled rate was $2.0 \%$ (95\% CI 1.0-3.3\%). There was no statistically significant heterogeneity $\left(I^{2}=0 \%\right.$; $P=0.7966$ ).

Four studies compared the rate of GEVB between patients who received and did not receive anticoagulation. Meta-analysis demonstrated that anticoagulation significantly decreased the rate of GEVB $(\mathrm{RR}=0.26$; $95 \% \mathrm{CI}$ $0.11-0.65 ; P=0.004)$. There was no statistically significant heterogeneity among studies $\left(I^{2}=0 \% ; P=0.74\right)$ (Fig. 3c).

\section{Death due to Bleeding Events}

Twenty-five studies reported the rate of death due to bleeding events after anticoagulation, and the pooled rate was $0.7 \% \quad(95 \%$ CI $0.3-1.3 \%)$. There was no statistically significant heterogeneity $\left(I^{2}=0 \% ; P>0.9999\right)$.

Five studies compared the rate of death due to bleeding events between patients who received and did not receive anticoagulation. Meta-analysis demonstrated that anticoagulation did not significantly influence the rate of death due to bleeding events $(\mathrm{RR}=0.26 ; 95 \% \mathrm{CI}$ $0.05-1.52 ; P=0.14)$. There was no statistically significant heterogeneity among studies $\left(I^{2}=0 \% ; P=0.93\right)$ (Fig. 3d).

\section{Overall Survival}

Six studies compared the overall survival rate between patients who received and did not receive anticoagulation. Meta-analysis demonstrated that anticoagulation significantly increased the overall survival rate $(\mathrm{RR}=1.11$; 95\% CI 1.03-1.21; $P=0.01)$. There was no statistically significant heterogeneity among studies $\left(I^{2}=0 \% ; P=0.47\right)$ (Fig. $\left.4 \mathrm{a}\right)$.

\section{One-Year Survival}

Two studies compared the 1-year survival rate between patients who received and did not receive anticoagulation. Meta-analysis demonstrated that anticoagulation did not significantly increase the 1-year survival rate $(\mathrm{RR}=1.19 ; 95 \%$ CI $0.65-2.20 ; P=0.57)$. There was a statistically significant heterogeneity among studies $\left(I^{2}=92 \% ; P=0.0006\right)$ (Fig. $\left.4 \mathrm{~b}\right)$.

\section{Three-Year Survival}

Two studies compared the 3-year survival rate between patients who received and did not receive anticoagulation. Meta-analysis demonstrated that anticoagulation did not significantly increase the 3-year survival rate $(\mathrm{RR}=1.21 ; 95 \%$ CI $0.78-1.87 ; P=0.40)$. There was a statistically significant heterogeneity among studies $\left(I^{2}=71 \% ; P=0.06\right)$ (Fig. $4 \mathrm{c}$ ).

\section{Five-Year Survival}

Two studies compared the 5-year survival rate between patients who received and did not receive anticoagulation. Meta-analysis demonstrated that anticoagulation did not significantly increase the 5-year survival rate $(\mathrm{RR}=1.08 ; 95 \%$ CI $0.97-1.21 ; P=0.16)$. There was no statistically significant heterogeneity among studies $\left(I^{2}=0 \% ; P=0.66\right)$ (Fig. $\left.4 \mathrm{~d}\right)$.

\section{Trend in Portal Vein Recanalization Rate After Anticoagulation According to the Patient Characteristics}

In the scattered plots, with an increase in the proportions of symptomatic PVT (abdominal pain and fever, new or worsening ascites, and GEVB), thrombus extension to SMV and/or SV, complete PVT, Child-Pugh class B and C, and HCC, platelet count, and serum creatinine (sCr) level, there is a decreasing trend in overall and complete portal vein recanalization rates (Supplementary Fig. 2). Spearman correlation analysis found that only HCC significantly correlated with a lower rate of complete portal 


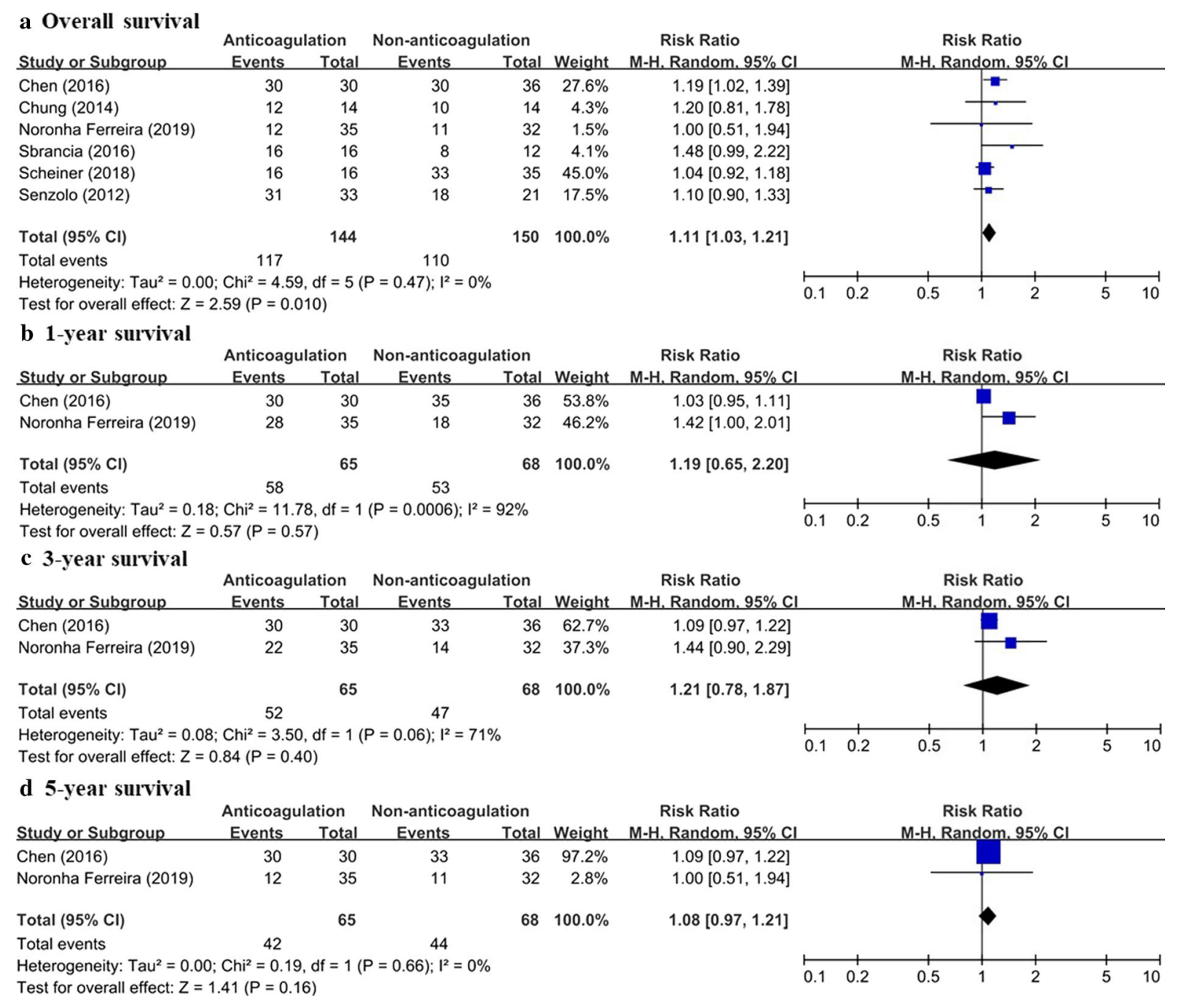

Fig. 4 Comparison of overall survival, 1-year survival, 3-year survival, and 5-year survival between anticoagulation and nonanticoagulation groups

vein recanalization $\left(P=0.037, r_{\mathrm{s}}=-0.90\right)$. We did not find any factor that significantly correlated with overall portal vein recanalization rate.

\section{Predictors of Portal Vein Recanalization}

Eight studies reported the predictors of portal vein recanalization after anticoagulation. The predictors evaluated included age, gender, previous portal hypertensive bleeding, ascites, hepatic encephalopathy, HCC, thrombophilia, isolated splenic vein thrombosis, isolated superior mesenteric vein thrombosis, complete PVT,
CTPV, platelet count, sCr level, international normalized ratio, Child-Pugh class and score, model for end-stage liver disease (MELD) score, interval between PVT diagnosis and initiation of anticoagulation, and duration of anticoagulation. Meta-analyses demonstrated that early initiation of anticoagulation ( $\mathrm{RR}=1.58 ; 95 \% \mathrm{CI}$ 1.21-2.07; $P=0.0007)$ and shorter duration of anticoagulation $\quad(\leq 6$ months versus $>6$ months $)(\mathrm{OR}=1.53 ; 95 \%$ CI 1.09-2.16; $P=0.02)$ significantly increased portal vein recanalization. Child-Pugh class $\mathrm{B}$ and $\mathrm{C}(\mathrm{RR}=$ $0.77 ; 95 \%$ CI $0.62-0.95 ; P=0.02$ ) and higher MELD score $(\mathrm{MD}=-1.48$; $95 \% \mathrm{CI}-2.20-0.76$; 
$P<0.0001)$ were significantly associated with decreased portal vein recanalization (Table 2).

\section{Predictors of Bleeding Events}

Two studies reported the predictors of bleeding events after anticoagulation. The predictors evaluated included type of anticoagulants (LMWH versus VKAs), Child-Pugh score, MELD score, platelet count, and duration of anticoagulation. Meta-analyses did not identify any predictor significantly associated with bleeding events after anticoagulation (Table 2).

\section{DISCUSSION}

Compared to the previous meta-analysis [11], our meta-analysis has several new findings. First, the pooled re-thrombosis rate after stopping anticoagulation was $46.7 \%$, suggesting the necessity of long-term anticoagulation in these patients. Second, we observed a significant benefit of anticoagulant therapy on the improvement of overall survival. Certainly, further validation is warranted. It remains uncertain about whether such an improvement is attributed to anticoagulation itself or portal vein recanalization as a response to anticoagulant therapy. Survival rate seems to be higher in patients achieving complete and partial portal vein recanalization than non-responders $[29,31,32]$. Third and most importantly, we were able to identify a series of predictors for portal vein recanalization, which may be useful to identify patients who are the most likely to benefit from anticoagulation.

Moreover, our meta-analysis has excluded the studies in which patients underwent splenectomy, splenic arterial embolization, TIPS, LT, and other major surgical interventions. Notably, such patients are different from cirrhotic patients with PVT who do not undergo any interventional procedure, since these procedures themselves can affect the progression of PVT and potentially confound the outcomes [52]. All relevant studies with and without a control group were included to calculate the rates of portal vein recanalization and bleeding. In addition, bleeding risk was classified as overall bleeding, major bleeding, UGIB, GEVB, and deaths due to bleeding events.

We found that among cirrhotic patients with PVT receiving anticoagulation, major bleeding accounted for less than a third of overall bleeding events. In other words, a majority of bleeding events in patients with liver cirrhosis and PVT while on anticoagulation are mild and may not require interruption of anticoagulant therapy. Due to a small number of bleeding events, we did not identify any factor associated with bleeding complications. In contrast to the traditional belief that anticoagulation increases bleeding risk in patients with portal hypertension, our meta-analysis indicated that anticoagulation was protective against UGIB and GEVB. A possible explanation for this phenomenon is that anticoagulant therapy may improve portal vein recanalization and then reduce portal vein pressure, thereby preventing bleeding from rupture of varicose veins and portal hypertensive gastropathy. Additionally, it should not be neglected that patients with active or recent GEVB and high-risk gastroesophageal varices were excluded in nearly all studies and that thorough screening for esophageal and/or gastric varices and prophylaxis of GEVB with either endoscopic band ligation or non-selective beta blockers were done prior to initiating anticoagulation.

The choice of anticoagulant drugs is inconsistent among the practice guidelines. The ACG guideline recommends unfractionated heparin and $\mathrm{LMWH}$ for the treatment of PVT in liver cirrhosis [6]. The Baveno VI consensus suggests that LMWH and VKAs appear to be equally effective in cirrhotic patients with PVT [7]. According to our subgroup analyses regarding the choices of anticoagulation regimes, overall recanalization rate was the highest in DOACs alone, followed by VKAs alone, fondaparinux alone, LMWH alone, LMWH in combination with antithrombin, and LMWH in continuation to VKAs; thrombus progression rate was the highest in LMWH in continuation to VKAs, followed by LMWH in combination with antithrombin, LMWH alone, DOACs alone, and VKA alone; bleeding rate was the highest in LMWH in continuation to VKAs, followed by fondaparinux alone, VKAs alone, and LMWH 
alone. Collectively, DOACs seemed to be more effective compared to LMWH and VKAs, which was consistent with the findings in non-cirrhotic PVT patients [53]. However, a higher rate of recanalization obtained by DOACs has been reported in only a single study [20]. In addition, the safety of DOACs is unclear in patients with advanced or decompensated cirrhosis [54]. Therefore, such a conclusion needs to be validated by a head-to-head randomized comparison. Except for clinical outcomes, the selection of anticoagulants should be also weighed according to the cost of drugs used, need of subcutaneous injection for LMWHs, and regular monitoring of INR in VKAs.

We found that the severity of PVT and underlying liver cirrhosis, inclusion of HCC, timing of initiation, and duration of anticoagulation might affect the benefits of anticoagulant therapy for PVT in liver cirrhosis. Completely occlusive PVT and thrombus extension to the SMV or SV seem to be associated with a lower portal vein recanalization rate. Compensated cirrhosis is associated with a higher portal vein recanalization rate; by comparison, patients with Child-Pugh class $\mathrm{B}$ and $\mathrm{C}$ and higher MELD score have a lower portal vein recanalization rate. Advanced liver cirrhosis has an aggravation of portal hypertension and a higher probability of using non-selective betablockers, thereby reducing the portal vein blood flow velocity which may contribute to the development and progression of PVT $[55,56]$. In addition, patients with advanced cirrhosis are more prone to the risk of thrombotic events and resistance to anticoagulation [57]. Advanced cirrhosis is associated with a reduction of hepatic folate storage and a suppression in its metabolic activation by hepatocytes, thereby causing secondary hyperhomocysteinemia, which is thought to contribute to thrombotic events [58]. Also, we found that the inclusion of HCC might affect portal vein recanalization. Although we excluded the studies involving only malignant PVT, some included studies still had a proportion of HCC patients, in whom the possibility of malignant PVT could not be completely excluded. Additionally, hypercoagulable state and tumor compression on the portal vein in HCC patients might lead to a low rate of portal vein recanalization. There seems to be an impact of a delay in starting anticoagulation on the recanalization of PVT in patients with and without cirrhosis $[59,60]$. Our meta-analysis confirmed that early initiation of anticoagulation increased portal vein recanalization. In addition, subgroup analysis found that complete portal vein recanalization rate seemed to be higher in studies excluding CTPV than those not excluding CTPV $\quad[49.3 \% \quad(95 \%) \quad$ CI $38.3 \%-60.4 \%) \quad$ versus $\quad 37.5 \% \quad(95 \% \quad$ CI $31.2 \%-43.9 \%)]$.

The recommended duration of anticoagulation for PVT in liver cirrhosis differs among the guidelines. The American Association for the Study of Liver Diseases guideline recommends anticoagulation for at least 3 months for all patients with acute PVT and long-term anticoagulation for patients with concomitant mesenteric vein thrombosis or those with permanent thrombotic risk factors [61]. The EASL and ACG guidelines recommend anticoagulation for at least 6 months in cirrhotic patients with PVT and consider lifelong anticoagulation in patients with thrombosis extending to SMV, those with a history suggestive of intestinal ischemia, or LT candidates $[6,8]$. Counter-intuitively, our meta-analysis found that a shorter duration of anticoagulation $(\leq 6$ months versus $>6$ months) significantly increased the rate of portal vein recanalization. This finding should be cautiously interpreted, because only two studies provided the relevant data. Additionally, both studies were observational in which continuing the use of anticoagulants was often dependent upon the dynamic assessment of PVT outcomes. In other words, anticoagulation would be more likely to be stopped earlier, if portal vein recanalization was achieved; by contrast, anticoagulation would be continued, if a thrombus remained unchanged. On the other hand, the duration of anticoagulation should also be based on the risk of re-thrombosis after stopping anticoagulation. However, this issue could not be evaluated, because the relevant data were lacking.

Our study has several limitations. (1) No randomized controlled trial has been identified yet, suggesting that the quality of evidence is 
relatively poor. (2) The characteristics of the study population, especially severity of cirrhosis, degree and extension of PVT, and CTPV, may affect the portal vein recanalization rates. Such data were heterogeneous among the included studies and were not reported in many studies. (3) Some studies did not exclude patients with HCC at baseline or during followup. (4) Some studies did not clarify the use of antiplatelet or thrombolytic therapy during anticoagulation. (5) Some studies did not clarify the type of anticoagulants. (6) The follow-up duration after anticoagulation varied among studies.

\section{CONCLUSION}

When the candidates for anticoagulation are carefully selected under adequate prophylaxis of variceal bleeding, anticoagulation is effective and safe for the treatment of PVT in cirrhosis. Additionally, anticoagulation may have a beneficial effect on survival in cirrhotic patients with PVT, but this impact of anticoagulation on survival should be further evaluated after adjusting for the severity of liver cirrhosis. Early initiation of anticoagulation can contribute to an increase in the rate of PVT recanalization. Child-Pugh class B and C and higher MELD score may be associated with lower recanalization rates of PVT. It may be useful to integrate these predictors into a scoring system to identify patients with liver cirrhosis and PVT who will not benefit from anticoagulant therapy.

\section{ACNOWLEDGEMENTS}

Funding. No funding or sponsorship was received for this study or publication of this article.

Authorship. All named authors meet the International Committee of Medical Journal Editors (ICMJE) criteria for authorship for this manuscript, take responsibility for the integrity of the work as a whole, and have given final approval for the version to be published.
Authorship Contributions. Conceptualization: Xingshun Qi; Methodology: Le Wang and Xingshun Qi; Validation: Xiaozhong Guo and Xingshun Qi; Formal analysis: Le Wang, Xiangbo $\mathrm{Xu}$, and Xingshun Qi; Investigation: Le Wang, Xiangbo Xu, and Xingshun Qi; Data curation: Le Wang, Xiangbo $\mathrm{Xu}$, and Xingshun Qi; Writing-original draft: Le Wang, Xiaozhong Guo, Xiangbo $\mathrm{Xu}$, and Xingshun Qi; Writing-review and editing: Le Wang, Xiaozhong Guo, Xiangbo Xu, Valerio De Stefano, Aurelie Plessier, Carlos Noronha Ferreira, and Xingshun Qi; Supervision: Xingshun Qi; Project administration: Xingshun Qi. All authors have made an intellectual contribution to the manuscript and approved the submission.

Disclosures. Le Wang, Xiaozhong Guo, Xiangbo Xu, Valerio De Stefano, Aurelie Plessier, Carlos Noronha Ferreira, and Xingshun Qi have nothing to disclose.

Compliance with Ethics Guidelines. This article is based on previously published studies and does not involve any new studies of human or animal subjects performed by any of the authors.

Data Availability. Full datasets generated during and/or analyzed during the current study are available from the corresponding author on reasonable request. Additional data are available in the supplementary materials.

Open Access. This article is licensed under a Creative Commons Attribution-NonCommercial 4.0 International License, which permits any non-commercial use, sharing, adaptation, distribution and reproduction in any medium or format, as long as you give appropriate credit to the original author(s) and the source, provide a link to the Creative Commons licence, and indicate if changes were made. The images or other third party material in this article are included in the article's Creative Commons licence, unless indicated otherwise in a credit line to the material. If material is not included in the article's Creative Commons licence and your intended use is not permitted by statutory regulation or exceeds the permitted use, you 
will need to obtain permission directly from the copyright holder. To view a copy of this licence, visit http://creativecommons.org/licenses/by$\mathrm{nc} / 4.0 /$.

\section{REFERENCES}

1. Intagliata NM, Caldwell SH, Tripodi A. Diagnosis, development, and treatment of portal vein thrombosis in patients with and without cirrhosis. Gastroenterology. 2019;156:1582-99.

2. Nery F, Chevret S, Condat B, et al. Causes and consequences of portal vein thrombosis in 1243 patients with cirrhosis: results of a longitudinal study. Hepatology. 2015;61:660-7.

3. Wang L, Xu X, Hou Y, Shao X, Guo X, Qi X. Acute mesenteric vein thrombosis after endoscopic injection sclerotherapy for esophageal varices in a patient with liver cirrhosis. Drug Discov Therap. 2019;13:118-21.

4. Ponziani FR, Zocco MA, Senzolo M, Pompili M, Gasbarrini A, Avolio AW. Portal vein thrombosis and liver transplantation: implications for waiting list period, surgical approach, early and late followup. Transplant Rev (Orlando). 2014;28:92-101.

5. Stine JG. Portal vein thrombosis, mortality and hepatic decompensation in patients with cirrhosis: a meta-analysis. World J Hepatol. 2015;7:2774.

6. Simonetto DA, Singal AK, Garcia-Tsao G, Caldwell SH, Ahn J, Kamath PS. ACG clinical guideline: disorders of the hepatic and mesenteric circulation. Am J Gastroenterol. 2020;115:18-40.

7. de Franchis R. Expanding consensus in portal hypertension: report of the Baveno VI consensus workshop: stratifying risk and individualizing care for portal hypertension. J Hepatol. 2015;63:743-52.

8. EASL Clinical Practice Guidelines. Vascular diseases of the liver. J Hepatol. 2016;64:179-202.

9. Qi X, Guo X, Yoshida EM, et al. Transient portal vein thrombosis in liver cirrhosis. BMC Med. 2018;16:83.

10. Qi X, De Stefano V, Li H, Dai J, Guo X, Fan D. Anticoagulation for the treatment of portal vein thrombosis in liver cirrhosis: a systematic review and meta-analysis of observational studies. Eur J Intern Med. 2015;26:23-9.

11. Loffredo L, Pastori D, Farcomeni A, Violi F. Effects of anticoagulants in patients with cirrhosis and portal vein thrombosis: a systematic review and meta-analysis. Gastroenterology. 2017;153(480-7): e1.

12. Naymagon L. Venous thrombosis of the liver: current and emerging concepts in management. Transl Res. 2020

13. Stang A. Critical evaluation of the Newcastle-Ottawa scale for the assessment of the quality of nonrandomized studies in meta-analyses. Eur J Epidemiol. 2010;25:603-5.

14. Chen H, Liu L, Qi X, et al. Efficacy and safety of anticoagulation in more advanced portal vein thrombosis in patients with liver cirrhosis. Eur J Gastroenterol Hepatol. 2016;28:82-9.

15. Hanafy AS, Abd-Elsalam S, Dawoud MM. Randomized controlled trial of rivaroxaban versus warfarin in the management of acute non-neoplastic portal vein thrombosis. Vascul Pharmacol. 2019;113: 86-91.

16. Hidaka H, Kokubu S, Sato T, et al. Antithrombin III for portal vein thrombosis in patients with liver disease: a randomized, double-blind, controlled trial. Hepatol Res. 2018;48:E107-16.

17. Cai M, Zhu K, Huang W, et al. Portal vein thrombosis after partial splenic embolization in liver cirrhosis: efficacy of anticoagulation and long-term follow-up. J Vasc Interv Radiol. 2013;24:1808-16.

18. Wan YM, Li YH, Wu HM, et al. Portal vein thrombosis before and after transjugular intrahepatic portosystemic shunt placement: an observational study (STROBE compliant). Medicine (Baltimore). 2017;96:e8498.

19. Wang Z, Jiang MS, Zhang HL, et al. Is Post-TIPS anticoagulation therapy necessary in patients with cirrhosis and portal vein thrombosis? A randomized controlled trial.

20. Nagaoki Y, Aikata H, Daijyo K, et al. Efficacy and safety of edoxaban for treatment of portal vein thrombosis following danaparoid sodium in patients with liver cirrhosis. Hepatol Res. 2018;48: 51-8.

21. Scheiner B, Stammet PR, Pokorny S, et al. Anticoagulation in non-malignant portal vein thrombosis is safe and improves hepatic function. Wien Klin Wochenschr. 2018;130:446-55.

22. Francoz C, Belghiti J, Vilgrain V, et al. Splanchnic vein thrombosis in candidates for liver transplantation: Usefulness of screening and anticoagulation. Gut. 2005;54:691-7. 
23. Amitrano L, Guardascione MA, Menchise A, et al. Safety and efficacy of anticoagulation therapy with low molecular weight heparin for portal vein thrombosis in patients with liver cirrhosis. J Clin Gastroenterol. 2010;44:448-51.

24. Delgado MG, Seijo S, Yepes I, et al. Efficacy and safety of anticoagulation on patients with cirrhosis and portal vein thrombosis. Clin Gastroenterol Hepatol. 2012;10:776-83.

25. Senzolo M, Rossetto V, et al. Prospective evaluation of anticoagulation and transjugular intrahepatic portosystemic shunt for the management of portal vein thrombosis in cirrhosis. Liver Int. 2012;32: 919-27.

26. Werner KT, Sando S, Carey EJ, et al. Portal vein thrombosis in patients with end stage liver disease awaiting liver transplantation: outcome of anticoagulation. Dig Dis Sci. 2013;58:1776-80.

27. Chung JW, Kim GH, Lee JH, et al. Safety, efficacy, and response predictors of anticoagulation for the treatment of nonmalignant portal-vein thrombosis in patients with cirrhosis: a propensity score matching analysis. Clin Mol Hepatol. 2014;20: 384-91.

28. Cui SB, Shu RH, Yan SP, et al. Efficacy and safety of anticoagulation therapy with different doses of enoxaparin for portal vein thrombosis in cirrhotic patients with hepatitis B. Eur J Gastroenterol Hepatol. 2015;27:914-9.

29. Fujiyama S, Saitoh S, Kawamura Y, et al. Portal vein thrombosis in liver cirrhosis: incidence, management, and outcome. BMC Gastroenterol. 2017;17: 112 .

30. Artaza T, Lopes M, Romero M, et al. Efficacy and safety of anticoagulation in non-malignant portal vein thrombosis in patients with liver cirrhosis. Gastroenterol Hepatol. 2018;41:611-7.

31. Kwon J, Koh Y, Yu SJ, Yoon JH. Low-molecularweight heparin treatment for portal vein thrombosis in liver cirrhosis: efficacy and the risk of hemorrhagic complications. Thromb Res. 2018;163:71-6.

32. La Mura V, Braham S, Tosetti G, et al. Harmful and beneficial effects of anticoagulants in patients with cirrhosis and portal vein thrombosis. Clin Gastroenterol Hepatol. 2018;16(1146-52):e4.

33. Bergere M, Erard-Poinsot D, Boillot O, et al. Portal vein thrombosis and liver cirrhosis: long-term anticoagulation is effective and safe. Clin Res Hepatol Gastroenterol. 2019;43:395-402.
34. Pettinari I, Vukotic R, Stefanescu H, et al. Clinical impact and safety of anticoagulants for portal vein thrombosis in cirrhosis. Am J Gastroenterol. $2019 ; 114: 258-66$

35. Rodriguez-Castro KI, Vitale A, Fadin M, et al. A prediction model for successful anticoagulation in cirrhotic portal vein thrombosis. Eur J Gastroenterol Hepatol. 2019;31:34-42.

36. Noronha Ferreira C, Reis D, Cortez-Pinto H, et al. Anticoagulation in cirrhosis and portal vein thrombosis is safe and improves prognosis in advanced cirrhosis. Dig Dis Sci. 2019;64:2671-83.

37. Hayashi T, Takatori H, Horii R, et al. Danaparoid sodium-based anticoagulation therapy for portal vein thrombosis in cirrhosis patients. BMC Gastroenterol. 2019;19:217.

38. Bento L, Huerta AR, Pascual C, et al. Antithrombotic therapy in non-neoplastic chronic portal venous thrombosis in cirrhosis: recanalization and liver function evaluation. Blood. 2011;1:18.

39. Butera G, Simone F, Iacò A, Calvaruso V, Di Marco $\mathrm{V}$, Craxì A. Anticoagulant treatment for not neoplastic portal vein thrombosis in patients with liver cirrhosis and esophageal varices. Digest Liver Dis. 2010;42:S37.

40. Caracciolo G, Garcovich M, Zocco MA, et al. Clinical outcome of partial portal vein thrombosis in cirrhotic patients: to observe or to treat? Digest Liver Dis. 2013;45:S171.

41. Takatori H, Hayashi T, Sunagozaka H, et al. Danaparoid sodium monotherapy for portal vein thrombosis in cirrhotic patients is as effective as combination therapy with antithrombin III. Hepatology. 2013;58:894A.

42. Risso A, Stradella D, Martini S, Rizzetto M, Salizzoni M. Liver transplantation in cirrhotic patients with portal vein thrombosis: a single centre experience. Digest Liver Dis. 2014;46:e40.

43. Cheruvathur P, Peter G, Mashhood V, Vinayakumar K, Sunil P. Portal vein thrombosis in patients with cirrhosis: outcome of anticoagulation. J Clin Exp Hepatol. 2015;5:S34-5.

44. Gheorghe L, Iacob S, Ester C, Iacob R, Popescu I, Gheorghe C. Safety and efficacy of anticoagulation therapy for splanhnic vein thrombosis in patients with liver cirrhosis on the waiting list for liver transplantation. Gastroenterology. 2015;148: S1063.

45. Inao M, Hirahara K, Sugawara K, Nakayama N, Imai Y, Mochida S. Usefulness of balloon-occluded retrograde obliteration (B-RTO) as a consolidation 
procedure after anticoagulation therapy in cirrhotic patients with portal vein thrombosis. Hepatology. 2015;62:935A.

46. Sbrancia M, Antonelli E, Bassotti G, Clerici C, Morelli O. Anticoagulation therapy for non malignant portal vein thrombosis in cirrhotic patients: a safe treatment? Digest Liver Dis. 2016;48:e82.

47. Tonon M, Piano S, Sacerdoti D, et al. Efficacy and safety of treatment of acute nonmalignant portal vein thrombosis with subcutaneous fondaparinux in patients with cirrhosis and marked thrombocytopenia. Digest Liver Dis. 2016;48:e25-6.

48. Tiwari LP, Venkateswaran AR, Rajkumar Solomon T, Mohamed KS, Kini R. Assessment of effect of anticoagulation for portal vein recanalization in non-tumoral portal vein thrombosis with cirrhosis. J Clin Exp Hepatol. 2018;8:S75-6.

49. Senzolo M, Piano S, Sacerdoti D, et al. Fondaparinux vs low molecular weight heparin in the treatment of non malignant portal vein thrombosis in patients cirrhosis. Digest Liver Dis. 2019;51:e42.

50. Tarantino G, Micucci G, Rupoli S, et al. Outcome of 119 cirrhotic patients with splanchnic thrombosis: a single center real-life experience. J Hepatol. 2019;70:e152.

51. Hayama K, Kaneko K, Tanabe T, et al. The add-on effect of AT-III in thrombolytic therapy for portal vein thrombosis. Gastroenterology. 2019;156:1348.

52. Qi X, Valla DC, Guo X. Anticoagulation for portal vein thrombosis in cirrhosis: selection of appropriate patients. Gastroenterology. 2018;154:760-1.

53. Naymagon L, Tremblay D, Zubizarreta N, et al. The efficacy and safety of direct oral anticoagulants in noncirrhotic portal vein thrombosis. Blood Adv. 2020;4:655-66.

54. Weinberg EM, Palecki J, Reddy KR. Direct-acting oral anticoagulants (DOACs) in cirrhosis and cirrhosis-associated portal vein thrombosis. Semin Liver Dis. 2019;39:195-208.

55. Xu X, Guo X, De Stefano V, et al. Nonselective betablockers and development of portal vein thrombosis in liver cirrhosis: a systematic review and metaanalysis. Hep Intl. 2019;13:468-81.

56. $\mathrm{Xu} \mathrm{X}, \mathrm{Xu} \mathrm{S}$, Primignani $\mathrm{M}$, et al. Nonselective $\beta$ blockers may progress the thrombosis of portal venous system in cirrhotic patients: a retrospective observational study. Adv Therapy. 2020;37: 1452-63.

57. Hemostatic balance in patients with liver cirrhosis: report of a consensus conference. Dig Liver Dis. 2016;48:455-467

58. Ventura P, Rosa MC, Abbati G, et al. Hyperhomocysteinaemia in chronic liver diseases: role of disease stage, vitamin status and methylenetetrahydrofolate reductase genetics. Liver Int. 2005;25:49-56.

59. Turnes J, Garcia-Pagan JC, Gonzalez M, et al. Portal hypertension-related complications after acute portal vein thrombosis: impact of early anticoagulation. Clin Gastroenterol Hepatol. 2008;6:1412-7.

60. Francoz C, Valla D, Durand F. Portal vein thrombosis, cirrhosis, and liver transplantation. J Hepatol. 2012;57:203-12.

61. DeLeve LD, Valla DC, Garcia-Tsao G. Vascular disorders of the liver. Hepatology. 2009;49:1729-64. 